\title{
A Family of Cubic Rational Maps and Matings of Cubic Polynomials
}

Mitsuhiro Shishikura and Tan Lei

\section{CONTENTS}

Introduction

1. Preliminaries

2. Statement of the Results and Examples

3. General Analysis on Branched Coverings and Matings

4. Proof of the Results: First Part

5. Proof of the Results: Second Part

Appendix: Matings Seen in Parameter Space and Some Numerical Observations

Acknowledgements

References
We study a family of cubic branched coverings and matings of cubic polynomials of the form $\mathrm{g} \Perp \mathrm{f}$, with $\mathrm{g}=\mathrm{g}_{\mathrm{a}}: \mathrm{z} \mapsto \mathrm{z}^{3}+\mathrm{a}$ and $f=P_{i}$ for $i=1,2,3$ or 4 . We give criteria for matability or not of critically finite $g_{a}$ with each $P_{i}$. The maps $g_{a} \Perp P_{1}$ illustrate features that do not occur for matings of quadratic polynomials: they never have Levy cycles but do sometimes have Thurston obstructions.

\section{INTRODUCTION}

A mating of two polynomials is a covering of the 2 -sphere constructed in a certain simple way, which gives it interesting dynamics.

The precise construction is as follows. Let $g$ and $f$ be two monic polynomials of the same degree, such that all critical points have finite orbits. We first add to $\mathbb{C}$ a circle of directions at infinity, and extend both $g$ and $f$ to this circle in the natural way. Next we sew up two copies of $\mathbb{C}$ along the circles at infinity, with opposite orientations. This gives a topological sphere. We then define a branched covering $g \Perp f$ that coincides with $g$ on one hemisphere and with $f$ on the other. If this map, or a suitable modification of it, is Thurston-equivalent to a rational map (which is to say, it is equivalent to a rational map up to isotopy relative to the postcritical set and topological conjugacy), we say that $g$ and $f$ are matable, and that $g \Perp f$ is a mating of $f$ and $g$.

In this paper we study matings of cubic polynomials $g \Perp f$ that occur in $\mathcal{A}$, the class of cubic branched coverings with one double critical point and one period-three orbit containing two simple critical points. By affine conjugation one can reduce to the case where $g$ is of the form of $g_{a}: z \mapsto z^{3}+a$ and $f$ is among four specific cubic polynomials $P_{1}$, 
$P_{2}, P_{3}$, and $P_{4}$ (Lemma 2.1). We want to study the matability of these pairs of polynomials.

There exist already several necessary or sufficient conditions for two general polynomials $g$ and $f$ to be matable, namely by means of Thurston obstructions and Levy cycles on one hand, and by means of the topology of $S^{2} / \sim_{\text {ray }}$ on the other hand, where $\sim_{\text {ray }}$ is the equivalence relation on the sewed up sphere generated by external rays of $g$ and $f$. We will introduce the notion of removable Levy cycles and formulate these various criteria.

Theorem 0.1. The four properties

(a) $g$ and $f$ are not matable,

(b) $g \Perp f$ has an irreducible obstruction which is not a removable Levy cycle,

(c) $g \Perp f$ has a non-removable Levy cycle,

(d) $S^{2} / \sim_{\text {ray }}$ is not homeomorphic to $S^{2}$

can be related as follows:

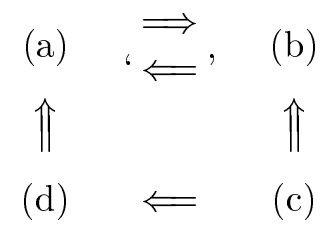

where $(\mathrm{a})^{\prime} \Longleftarrow$ '(b) means that $(\mathrm{a}) \Longleftarrow$ (b) if a suitable modification $s(g \Perp f)$ of $g \Perp f$ (see Theorem 3.12) has a hyperbolic orbifold (there are counterexamples otherwise).

Implication $(\mathrm{c}) \Longrightarrow(\mathrm{b})$ is trivial, and $(\mathrm{d}) \Longrightarrow(\mathrm{a})$ was proved by M. Rees (see also [Shishikura 2000]). We will give a proof of the rest; other similar proofs exist in the literature.

We first show how to modify a branched covering in order to delete a maximal number of removable Levy cycles, without changing the dynamics too much (Theorem 3.12 and Corollary 3.13). We then apply this technique to matings, making use of the ray-equivalence relation (Proposition 3.16).

For quadratic matings and cubic matings related to Newton maps, we also have (b) $\Longrightarrow$ (c) [Tan 1992; 1997]; therefore in this case the four conditions are in fact equivalent.

One major objective of this work is to show that (b), (c), and (d) are not always equivalent (K. Pilgrim [1994] has found other examples of this type). More precisely, we show that (a) $\Leftrightarrow$ (b) for a mating $g \Perp f$ in $\mathcal{A}$ and that, for $g_{a} \Perp P_{1}, S^{2} / \sim_{\text {ray }}$ is a sphere, there is no Levy cycles and there are $a$ values for which $g_{a} \Perp P_{1}$ satisfies (b) (and therefore (a)). We conjecture that $g_{a}$ and $P_{1}$ are not matable if and only if for $s=a$ or $\bar{a}$, the two external rays $R_{g_{s}}\left(\frac{7}{26}\right)$ and $R_{g_{s}}\left(\frac{9}{26}\right)$ land at the same point. The set of such $s$ values form a sublimb of the connectedness locus $M_{3}$. So far we can only prove that for $s$ in one half of the sublimb union the largest copy of $M_{3}$ in the sublimb, and $a=s$ or $\bar{s}, g_{a}$ and $P_{1}$ are not matable. We find also $a$ values outside of the sublimb (in terms of the topological entropy of $g_{a}$ on its Hubbard tree) for which $g_{a}$ and $P_{1}$ are matable. But we are unable to give a complete proof of either direction of the conjecture. It might therefore not be a realistic conjecture.

For each other choice of $f$, we try also to find conditions on $a$ so that $g_{a}$ and $f$ are matable. Two of the four possible candidates for $f$, denoted by $P_{3}$ and $P_{4}$, are non real polynomials with star-like Hubbard trees. We shall prove that each of them is matable with any critically finite $g_{a}$ (Theorem 2.6). For $g_{a} \Perp P_{2}$, the remaining case, we show that (c) is equivalent to the fact that the two external rays $R_{g_{a}}\left(\frac{5}{8}\right)$ and $R_{g_{a}}\left(\frac{7}{8}\right)$ land at the same point (Theorem 2.5). We conjecture that this last condition is a consequence of (b), therefore the four properties (a), (b), (c), and (d) are equivalent.

In order to prove these results, we study at first the structure of Thurston obstructions for a general map in $\mathcal{A}$ (Theorem 2.2 and Proposition 2.3), and then apply this to matings, using techniques about Hubbard trees, external rays, etc.

Although our results are only partial, some of the tools introduced here might be useful for the study of other class of maps. We emphasise also that most phenomena about matings in $\mathcal{A}$ were first observed by computer experiments, and then proved or partially proved mathematically later on. Here the computer experiments have served us as a guide to rigorous mathematical results.

The paper is organized as follows: Sections $1 \mathrm{~A}-1 \mathrm{C}$ recall definitions and results. In Section 2 we introduce the class $\mathcal{A}$ and the polynomials $P_{i}$, state our results about structures of Thurston obstructions for a map in $\mathcal{A}$ and, for each $i$, give criteria for $g_{a}$ and $P_{i}$ to be not matable. We give also an example of a mating in $\mathcal{A}$ which has a Thurston obstruction but no Levy cycles. Section 3A contains a sufficient 
condition for $S^{2} / \sim_{\text {ray }}$ of a mating to be a sphere. In Sections 3B-3H we review and develop several techniques for the analysis of Thurston obstructions and (removable) Levy cycles together with their applications to matings. In Section 4 we prove our criteria about non-matability of $g_{a}$ and $P_{i}$ for $i=2,3,4$ and show that for $g_{a} \Perp P_{1}$, the quotient $S^{2} / \sim_{\text {ray }}$ is a sphere. In Section 5 we establish results regarding Thurston obstructions for a map in $\mathcal{A}$ and for $g_{a} \Perp P_{1}$.

In the appendix, we give interpretations of our results in the parameter space of rational maps in $\mathcal{A}$ along with several numerical observations.

\section{PRELIMINARIES}

\section{A. Thurston and Levy's Theory on Branched Coverings from $S^{2}$ to $S^{2}$}

All branched coverings in this paper are assumed to be orientation-preserving and of degree greater than one.

Definition (postcritical set). Let $F: S^{2} \rightarrow S^{2}$ be a branched covering of degree $d$. Set

$\Omega_{F}=\{$ critical points of $F\}$ and $P_{F}=\bigcup_{n>0} F^{n}\left(\Omega_{F}\right)$.

The set $P_{F}$ is called the postcritical set of $F$. We say that $F$ is postcritically finite, if $P_{F}$ is finite.

\section{Definition (equivalence between branched coverings).}

We say two postcritically finite branched coverings $F$ and $G$ from $S^{2}$ to $S^{2}$ are equivalent, and write $F \sim$ $G$, if some topological conjugate of $G$ is isotopic to $F$ rel $P_{F}$. More precisely, there exist two orientationpreserving homeomorphisms $\theta_{1}, \theta_{2}: S^{2} \rightarrow S^{2}$ such that $G \circ \theta_{1}=\theta_{2} \circ F, \theta_{1}=\theta_{2}$ on $P_{F}, \theta_{i}\left(P_{F}\right)=P_{G}$ for $i=1,2$, and $\theta_{1}, \theta_{2}$ are isotopic relative to $P_{F}$.

There are some equivalent conditions for $F, G$ to be equivalent. First, we summarize related results of Thurston and Levy. For details and proofs see [Thurston 1983; Douady and Hubbard 1993].

Definition (multicurve). Let $F: S^{2} \rightarrow S^{2}$ be a postcritically finite branched covering, with degree $d \geq 2$. A simple closed curve in $S^{2}-P_{F}$ is called peripheral if it bounds a disc containing at most one point of $P_{F}$. A multicurve $\Gamma$ is a collection of finitely many disjoint nonperipheral simple closed curves in $S^{2}-P_{F}$ such that no two curves are isotopic to each other in $S^{2}-P_{F}$.

For a multicurve $\Gamma$ of $F$, the Thurston linear transformation $F_{\Gamma}$ is a linear map from $\mathbb{R}^{\Gamma}=\left\{\sum_{\gamma \in \Gamma} c_{\gamma} \gamma \mid\right.$ $\left.c_{\gamma} \in \mathbb{R}\right\}$ to itself defined by

$$
F_{\Gamma}(\gamma)=\sum_{\gamma^{\prime} \subset F^{-1}(\gamma)} \frac{1}{\operatorname{deg}\left(F: \gamma^{\prime} \rightarrow \gamma\right)}\left[\gamma^{\prime}\right]_{\Gamma} \quad \text { for } \gamma \in \Gamma,
$$

where the sum is over all connected components $\gamma^{\prime}$ of $F^{-1}(\gamma)$, and $\left[\gamma^{\prime}\right]_{\Gamma}$ denotes the curve in $\Gamma$ isotopic to $\gamma^{\prime}$ if it exists and $\left[\gamma^{\prime}\right]_{\Gamma}=0$ otherwise. We denote by $\lambda_{\Gamma}$ the leading eigenvalue of $F_{\Gamma}$.

A multicurve $\Gamma$ with $\lambda_{\Gamma} \geq 1$ is called a Thurston obstruction. Initially, Thurston obstructions are defined for $F$-invariant multicurves. But the two definitions are equivalent, as shown in Lemma 3.5 below.

Theorem 1.1 (Thurston). Suppose $F: S^{2} \rightarrow S^{2}$ is a postcritically finite branched covering. If $F$ has no Thurston obstructions then $F$ is equivalent to a rational map. The rational map is unique up to conformal conjugacy. If $F$ has a hyperbolic orbifold (see the remark below) and Thurston obstructions, then $F$ is not equivalent to a rational map.

Remark. The notion of orbifold can be found in [Douady and Hubbard 1993]. If a branched covering $F$ is has a non-hyperbolic orbifold, then $F^{-1}\left(P_{F}\right) \subset$ $\Omega_{F} \cup P_{F}$ and $\# P_{F} \leq 4$.

Definition (good, degenerate and removable Levy cycles). A multicurve $\Gamma=\left\{\gamma_{1}, \ldots, \gamma_{n}\right\}$ of $F$ is called a Levy cycle if each $F^{-1}\left(\gamma_{i+1}\right)$ contains a component $\gamma_{i+1}^{\prime}$ isotopic in $S^{2}-P_{F}$ to $\gamma_{i}$ and $F: \gamma_{i+1}^{\prime} \rightarrow \gamma_{i+1}$ is of degree one for $i=0, \ldots, n-1$, where $\gamma_{0}=\gamma_{n}$. We say that $\Gamma$ is a good Levy cycle if the connected components of $S^{2}-\bigcup_{i=1}^{n} \gamma_{i}$ are $B_{1}, B_{2}, \ldots, B_{m}, C$, with $B_{i}$ discs, and, in case $n=1, C=\varnothing$ and $F: \gamma_{1}^{\prime} \rightarrow \gamma_{1}$ reverses the orientation; in case $n>1$, one component $C^{\prime}$ of $F^{-1}(C)$ is isotopic to $C$ and $F: C^{\prime} \rightarrow C$ is of degree one. We say that $\Gamma$ is a degenerate Levy cycle if the connected components of $S^{2}-\bigcup_{i=1}^{n} \gamma_{i}$ are $B_{1}, B_{2}, \ldots, B_{n}, C$, with $B_{i}$ discs, and each $F^{-1}\left(B_{i+1}\right)$ has a component $B_{i+1}^{\prime}$ isotopic to $B_{i}\left(\right.$ rel $\left.P_{F}\right)$, and $F: B_{i+1}^{\prime} \rightarrow B_{i+1}$ is of degree one for $i=0,1, \ldots, n-1$, where $B_{0}=B_{n}$. We say that $\Gamma$ is a removable Levy cycle if it is degenerate and 
for all $j \geq 1$ and all $i$, the components of $F^{-j}\left(B_{i}\right)$ are discs.

Here are some known results concerning degree two mappings:

Theorem 1.2 [Levy 1985]. Suppose $F$ is of degree 2 and postcritically finite. If it has a Thurston obstruction then it has a Levy cycle.

Theorem 1.3 [Rees 1986; Tan 1992]. Suppose F is of degree 2 and postcritically finite. If it has a nonremovable Levy cycle then it has a good Levy cycle.

We will see in this work many counterexamples of Theorem 1.2 for higher-degree mappings. In fact we are going to construct a family of cubic branched coverings which have nested obstructions (see definition below) but no Levy cycles. A counterexample of Theorem 1.3 can be easily found.

We will see in Section $3 \mathrm{E}$ that each removable Levy cycle can be literally 'removed' by modifying the map. On the other hand, one can not always remove simultaneously two such cycles if they are not disjoint. The following result, which will be made precise and proved in Section 3E, gives the condition under which one can remove all the Levy cycles and get a rational map:

Definition (irreducible obstruction). We say that a multicurve $\Gamma$ is an irreducible obstruction if the matrix $F_{\Gamma}$ is irreducible with $\lambda(\Gamma) \geq 1$.

Theorem and definition (weak equivalence). We say that $F$ is weakly equivalent to a rational map if all irreducible obstructions of $F$ are removable Levy cycles smaller or equal to an element of $\Sigma^{\prime}(F)$ (see Section $3 \mathrm{E}$ for definition). In this case a suitable modification of $F$ is equivalent to a rational map.

Definition (nested obstruction). A multicurve $\Gamma$ for a postcritically finite map $F$ is a nested obstruction if $S^{2}-\Gamma$ has only two disc components and $\lambda\left(F_{\Gamma}\right) \geq 1$. For example a Levy cycle with one or two curves is a nested obstruction.

\section{B. Matings of Polynomials}

In this section, we give definitions of matings.

Definition (formal mating). Let $f$ and $g$ be two monic polynomials of degree $d$. Set

$$
\tilde{\mathbb{C}}=\mathbb{C} \cup\left\{\infty \cdot e^{2 \pi i s} \mid s \in \mathbf{T}=\mathbb{R} / \mathbb{Z}\right\} .
$$

We then extend $f$ and $g$ continuously to $\tilde{\mathbb{C}}$ by setting

$$
\begin{aligned}
& f\left(\infty \cdot e^{2 \pi i s}\right)=\infty \cdot e^{2 d \pi i s}, \\
& g\left(\infty \cdot e^{2 \pi i s}\right)=\infty \cdot e^{2 d \pi i s} .
\end{aligned}
$$

Set

$$
S_{f, g}^{2}=\tilde{\mathbb{C}}_{f} \sqcup \tilde{\mathbb{C}}_{g} /\left\{\left(\infty \cdot e^{2 \pi i s}, f\right) \approx\left(\infty \cdot e^{-2 \pi i s}, g\right)\right\} .
$$

The formal mating of $f$ and $g$ is defined to be the branched covering $f \Perp g: S_{f, g}^{2} \rightarrow S_{f, g}^{2}$ with

$$
f \Perp g=f \text { on } \tilde{\mathbb{C}}_{f} \text { and } f \Perp g=g \text { on } \tilde{\mathbb{C}}_{g} .
$$

When there is no ambiguity, we write $S^{2}$ instead of $S_{f, g}^{2}$.

Definition (matability). We say that two monic postcritically finite degree $d$ polynomials $f$ and $g$ are matable if $f \Perp g$ is weakly equivalent to a rational map.

See [Douady and Hubbard 1984] or [Carleson and Gamelin 1993] for the definitions of the Julia set $J_{f}$, the filled-in Julia set $K_{f}$ and the external rays of a polynomial $f$. Recall that if $f$ is postcritically finite, then $K_{f}$ is connected and locally connected, and all external rays land at $K_{f}$. For $\theta \in \mathbf{T}$, let us denote by $R_{f}(\theta)$ the closure in $\tilde{\mathbb{C}}_{f}$ of the external ray of angle $\theta$.

In $S_{f, g}^{2}$, the external rays $R_{f}(\theta)$ and $R_{g}(-\theta)$ are connected at the point $\left(\infty \cdot e^{2 \pi i \theta}, f\right)$.

Definition (ray-equivalence). For $x$ and $y$ in $\tilde{\mathbb{C}}_{f}$, we define $\sim_{f}$ to be the equivalence relation generated by $x, y \in R_{f}(\theta)$ for some $\theta$. The relation $\sim_{g}$ on $\tilde{\mathbb{C}}_{g}$ is defined similarly. In $S_{f, g}^{2}$, define the ray-equivalence $\sim_{\text {ray }}$ to be the equivalence relation generated by $\sim_{f}$ on $\tilde{\mathbb{C}}_{f}$ and $\sim_{g}$ on $\tilde{\mathbb{C}}_{g}$. Denote by $[x]$ the rayequivalence class (or for short: the ray class) of $x \in S^{2}$.

A Levy cycle for a mating is closely related to a periodic cycle of ray classes.

Theorem 1.4 [Tan 1992]. Set $F=f \Perp g . \quad$ Each Levy cycle $\Gamma$ corresponds to a unique periodic cycle of ray classes $\left[x_{0}\right], \ldots,\left[x_{m-1}\right]$ (they are called limit set of $\Gamma$ ) and a finitely connected subset $X_{i}$ of $\left[x_{i}\right]$ for each $i$ such that $F:\left[x_{i}\right] \rightarrow\left[x_{i+1} \bmod m\right]$, $X_{i} \rightarrow X_{i+1} \bmod m$ are homeomorphisms, and $\Gamma$ is isotopic to the boundary of a tubular neighborhood of $X_{0} \cup \cdots \cup X_{m-1}$. In particular, 
1. if $\Gamma$ is degenerate, then

$$
\#\left[x_{i}\right] \cap P_{F} \geq \# X_{i} \cap P_{F} \geq 2,
$$

and each component of $X_{i}$ is a tree;

2. if $\Gamma$ is not degenerate, each $X_{i}\left(\right.$ and $\left.\left[x_{i}\right]\right)$ contains a closed curve;

3. if $\Gamma$ is a good, then $X_{0}$ is connected, $F\left(X_{0}\right)=X_{0}$, $F\left(\left[x_{0}\right]\right)=\left[x_{0}\right]$. If $\# \Gamma>1$, then $\pi_{1}\left(X_{0}\right)$ is isomorphic to the fundamental group of the unique nondisc component of $S^{2}-\Gamma$. If $\# \Gamma \leq 2$, then $X_{0}$ is a simple closed curve, $F: X_{0} \rightarrow X_{0}$ reverses the orientation and $X_{0}$ contains exactly two fixed points of $F$ (if $F$ is quadratic, $\left[x_{0}\right]$ contains exactly two fixed points, even when $\# \Gamma>2$ ).

\section{C. M. Rees' Theory of Matings}

Denote by $\gamma_{f}(\theta)$ the landing point of $R_{f}(\theta)$ on $J_{f}$. Note that

$$
S_{f, g}^{2} / \sim_{\text {ray }}=K_{f} \sqcup K_{g} /\left\{\gamma_{f}(\theta) \approx \gamma_{g}(-\theta)\right\} .
$$

Theorem 1.5 [Rees 1992; Shishikura 2000]. Suppose $f$ and $g$ are two monic postcritically finite polynomials, both with degree $d$. If $f$ and $g$ are matable then the following two conditions are satisfied:

(i) the quotient space $S_{f, g}^{2} / \sim_{\text {ray }}$ is homeomorphic to $S^{2}$;

(ii) the quotient map

$$
[f \Perp g]: S_{f, g}^{2} / \sim_{\text {ray }} \rightarrow S_{f, g}^{2} / \sim_{\text {ray }}
$$

is topologically conjugate to a rational map.

This theorem is presented and proved in case of $d=2$ and $f$ and $g$ both hyperbolic polynomials in [Rees 1992]. Shishikura gave a proof of the general case in [Shishikura 2000]. Note that a ray class of $f \Perp g$ containing at least two postcritical points will represent one postcritical point for the quotient mapping. This is the reason for which we have to modify the formal mating in order to study the equivalence between a mating and a rational map.

Condition (i) is a purely topological one, but the condition (ii) involves conformal structure problems. For quadratic matings and matings related to cubic Newton methods, (i) implies also the matability of the polynomials [Rees 1986; Tan 1992; 1997]. But our study in this paper will show that this is not always true.

\section{STATEMENT OF THE RESULTS AND EXAMPLES}

\section{A. The Class $\mathcal{A}$ and Related Matings}

We are mainly interested in a special class $\mathcal{A}$ of cubic branched coverings and related matings.

Notation. We denote by $\mathcal{A}$ the set of cubic branched coverings $F$ satisfying: $F$ has a double critical point $w$ and a period-three cycle $x \mapsto y \mapsto z \mapsto x$ containing two simple critical points $x$ and $y$. Denote by $a$ the critical value $F(w)$.

We want to study matings in $\mathcal{A}$. It turns out that they all have a simple form, as indicated in the next lemma. First note that cubic polynomials with a double critical point are always of the form $g_{a}: z \mapsto$ $z^{3}+a$. Next, there are exactly four pairs of monic cubic polynomials $P_{1}, \tilde{P}_{1}, P_{2}, \tilde{P}_{2}, P_{3}, \tilde{P}_{3}, P_{4}, \tilde{P}_{4}$ for which there is a 3-periodic orbit containing two simple critical points, with $\tilde{P}_{i}(t)=-P_{i}(-t)$ for all $t \in \mathbb{C}$. Denote by $x, y$ the two simple critical points with orbit $x \mapsto y \mapsto z \mapsto x$. We choose $P_{1}$ to be a monic real cubic polynomial with $x, y, z$ real and $y<$ $x<z$. These properties determine $P_{1}$ uniquely up to translation by a real vector. We choose $P_{2}$ to be a monic cubic polynomial for which the imaginary axis $i \mathbb{R}$ is preserved, $x, y, z \in i \mathbb{R}$ and $i z<i y<i x$. For $P_{2}$, the fixed point $\alpha^{\prime} \in[y, z]$ has external angles $\frac{1}{8}, \frac{3}{8}$.

An efficient way to describe a polynomial is by its Hubbard tree. According to Douady and Hubbard, each postcritically finite monic polynomial $g$ has a forward invariant Hubbard tree, which is the convex hull of $P_{g}$ in $K_{g}$ [Douady and Hubbard 1984; 1985; Poirier 1993]. The Hubbard tree of $P_{1}, \ldots, P_{4}$ are given in Figure 1.

Lemma 2.1. The matings in $\mathcal{A}$ are exactly maps of the form $g \Perp f$, with $g=g_{a}: z^{3}+a$ and $f \in$ $\left\{P_{i}, \tilde{P}_{i}, i=1,2,3,4\right\}$. Moreover each mating is topologically conjugate to $g_{a} \Perp f$, for some value a and $f \in\left\{P_{1}, P_{2}, P_{3}, P_{4}\right\}$.

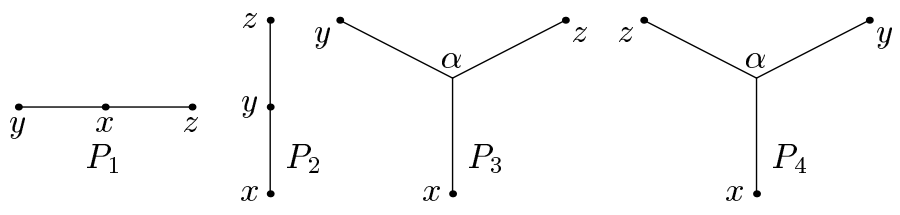

FIGURE 1. The Hubbard trees of $P_{1}, P_{2}, P_{3}, P_{4}$. 
Proof. Clearly the branched coverings of the form $g_{a} \Perp P_{i}$ and $g_{a} \Perp \tilde{P}_{i}$ belong to $\mathcal{A}$. On the other hand, if a mating $g \Perp f$ is in $\mathcal{A}$, one of the polynomials, say, $f$, must have a 3 -periodic orbit containing two simple critical points, and the other polynomial, $g$, must have a double critical point. So $g$ is of the form $z^{3}+a$ and $f$ is among $P_{i}$ and $\tilde{P}_{i}$. We notice also that $g_{a} \Perp \tilde{P}_{i}$ is conjugate to $g_{-a} \Perp P_{i}$.

\section{B. Results}

In order to study which matings in $\mathcal{A}$ are equivalent to rational maps, we need to study at first possible obstructions for a general map in $\mathcal{A}$. Here are our results:

Theorem 2.2. Let $\Gamma$ be an irreducible obstruction for a postcritically finite map $F$ in $\mathcal{A}$. Then exactly one of the following statements holds.

1. $\Gamma$ is a removable Levy cycle.

2. Some preimages of $\Gamma$ contains a good Levy cycle with at most two elements.

3. $\Gamma$ is a nested obstruction but not a Levy cycle, with $a$ and $y$ contained in one disc component, say $D_{+}(\Gamma)$, of $S^{2}-\Gamma$, $z$ in the other disc component $D_{-}(\Gamma)$ of $S^{2}-\Gamma$ and $x \notin D_{+}(\Gamma) \cup D_{-}(\Gamma)$.

Proposition 2.3. Assume that $\Gamma^{\prime}$ is a multicurve for a postcritically finite map $F$ in $\mathcal{A}$, such that $S^{2}-\Gamma^{\prime}$ has only two disc components $D_{+}\left(\Gamma^{\prime}\right)$ and $D_{-}\left(\Gamma^{\prime}\right)$ with $a, y \in D_{+}\left(\Gamma^{\prime}\right), z \in D_{-}\left(\Gamma^{\prime}\right)$ and $x \notin D_{+}\left(\Gamma^{\prime}\right) \cup D_{-}\left(\Gamma^{\prime}\right)$.

For any $\Gamma$ a submulticurve of $\Gamma^{\prime}$, denote by $D_{ \pm}(\Gamma)$ the disc component of $S^{2}-\Gamma$ containing $D_{ \pm}\left(\Gamma^{\prime}\right)$. Set $A_{0}(\Gamma)=F^{-1}\left(D_{+}(\Gamma)\right)$.

Then $\Gamma^{\prime}$ is a Thurston obstruction if and only if there is a submulticurve $\Gamma$ of $\Gamma^{\prime}$ such that, up to isotopy, $D_{+}(\Gamma)$ is contained in a component of $F^{-1}\left(D_{-}(\Gamma)\right), A_{0}(\Gamma)$ contains no curve of $\Gamma$ and separates $D_{+}(\Gamma)$ and $D_{-}(\Gamma)$, and $D_{-}(\Gamma)$ is separated from $A_{0}(\Gamma)$ by a component of $F^{-1}\left(\partial A_{0}(\Gamma)\right)$. In this case $\lambda_{\Gamma}=1$ and $\mu_{\Gamma}=\frac{1}{2}(1+\sqrt{5})$, where $\mu_{\Gamma}$ is the leading eigenvalue of the unweighted Thurston linear transformation defined by

$$
F_{\#, \Gamma}: \gamma \mapsto \sum_{\gamma^{\prime} \subset F^{-1}(\gamma)}\left[\gamma^{\prime}\right]_{\Gamma} \quad \text { for } \gamma \in \Gamma .
$$

We now turn to results and conjectures about matings. It is a case by case study. We write $\theta \sim_{g_{a}} \eta$ if the two external rays for $g_{a}$ of angles $\theta$ and $\eta$ land at the same point.
Theorem 2.4. Let $g_{a}$ be postcritically finite. Then $S_{g_{a}, P_{1}}^{2} / \sim_{\text {ray }}$ is homeomorphic to $S^{2}$. Moreover, of the following conditions, 1 and 2 are equivalent, 3 implies 1 and 4 , and both 1 and 4 imply 5.

1. $g_{a}$ and $P_{1}$ are not matable.

2. $g_{a} \Perp P_{1}$ has a nested obstruction which is not a Levy cycle.

3. For $\beta_{0}$ the fixed point of $g_{a}$ with external angle 0 , either $\beta_{0}$ and $g_{a}(a)$ are in the same component of $J_{a}-\{w\}$ and $g_{a}^{3}$ is renormalizable (i.e. $g_{a}^{3}$ is a cubic polynomial-like mapping with connected Julia set $)$, or $\beta_{0}, g_{a}(a)$ and $g_{a}^{2}(a)$ are in the same component of $J_{a}-\{w\}$.

4. Either $\frac{7}{26} \sim_{g_{a}} \frac{9}{26}$ or $\frac{19}{26} \sim_{g_{a}} \frac{17}{26}$.

5. The topological entropy of $g_{a}$ on its Hubbard tree is at least $\log \left(\frac{1}{2}(1+\sqrt{5})\right)$.

We conjecture that 1 is equivalent to 4 . We consider the above theorem as a partial proof for the direction $4 \Longrightarrow 1$ and the direction $4 \Longleftarrow 1$. At present we don't have a complete proof of either direction of the conjecture.

The part $1 \Longrightarrow 5$ relies on a recent result of Shishikura relating $\mu_{\Gamma}$ to the topological entropy. It is easy to see from examples that 5 does not imply 4 .

In the appendix we give parametric interpretations for Conditions 3 and 4.

Theorem 2.5. Let $g_{a}$ be postcritically finite. Then conditions $\mathrm{B}, \mathrm{C}$, and $\mathrm{D}$ are equivalent and each implies A.

A. $g_{a}$ and $P_{2}$ are not matable.

B. $\frac{5}{8} \sim g_{a} \frac{7}{8}$.

C. $g_{a} \Perp P_{2}$ has a good Levy cycle with at most two elements.

D. $g_{a} \Perp P_{2}$ has a non-removable Levy cycle.

We also conjecture that $\mathrm{A}$ and $\mathrm{B}$ are equivalent.

Theorem 2.6. If $g_{a}$ is postcritically finite, then $g_{a}$ and $P_{i}$ are matable, for $i=3,4$.

\section{C. An Example of a Nested Obstruction}

Consider $g=g_{c}: z \mapsto z^{3}+c$ such that the double critical point $w=0$ has orbit $w \mapsto c \mapsto c^{3}+c \mapsto w$ and that the two rays $R_{g}\left(\frac{7}{26}\right)$ and $R_{g}\left(\frac{9}{26}\right)$ land at the same point. These conditions determine $c$ uniquely. 


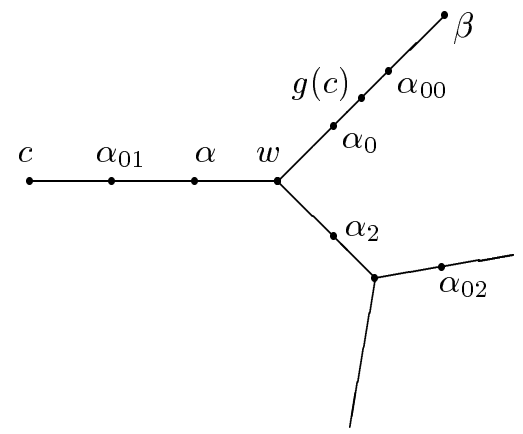

FIGURE 2. Extended Hubbard tree for $g$.

Figure 2 shows an extension of the Hubbard tree of $g$. The points $\alpha$ and $\beta$ are fixed points,

$$
\left\{\alpha, \alpha_{0}, \alpha_{2}\right\}=g^{-1}(\alpha),
$$

and $\left\{\alpha_{01}, \alpha_{00}, \alpha_{12}\right\}=g^{-1}\left(\alpha_{0}\right)$. The external angles of these points are

$$
\begin{gathered}
>\beta=\{0\}, \\
>\alpha=\left\{\frac{1}{8}, \frac{3}{8}\right\},>\alpha_{0}=\left\{\frac{1}{24}, \frac{19}{24}\right\},>\alpha_{2}=\left\{\frac{11}{24}, \frac{17}{24}\right\}, \\
>\alpha_{01}=\left\{\frac{19}{72}, \frac{25}{72}\right\},>\alpha_{00}=\left\{\frac{1}{72}, \frac{67}{72}\right\},>\alpha_{02}=\left\{\frac{43}{72}, \frac{49}{72}\right\} .
\end{gathered}
$$

Figure 3 shows an extension of the Hubbard tree of $P_{1}$ (see also Figure 1). Here $\beta^{\prime}, \beta^{\prime \prime}$ and $\alpha^{\prime}$ are fixed points, $\left\{\beta^{\prime}, \beta_{1}^{\prime}, \beta_{2}^{\prime}\right\}=P_{1}^{-1}(\beta),\left\{\alpha^{\prime}, \alpha_{0}^{\prime}, \alpha_{2}^{\prime}\right\}=$ $P_{1}^{-1}\left(\alpha^{\prime}\right),\left\{\alpha_{01}^{\prime}, \alpha_{00}^{\prime}, \alpha_{02}^{\prime}\right\}=P_{1}^{-1}\left(\alpha_{0}^{\prime}\right)$ and $x_{*}, y_{*}, z_{*}$ are the roots of basins of attraction of $x, y, z$, respectively (i.e. repelling periodic orbit of period 3 on the boundary of the basins). The external angles of these points are

$>\beta^{\prime}=\{0\},>\beta^{\prime \prime}=\left\{\frac{1}{2}\right\},>\beta_{1}^{\prime}=\left\{\frac{1}{3}\right\},>\beta_{2}^{\prime}=\left\{\frac{2}{3}\right\}$, $>x_{*}=\left\{\frac{3}{13}, \frac{10}{13}\right\},>y_{*}=\left\{\frac{4}{13}, \frac{9}{13}\right\},>z_{*}=\left\{\frac{1}{13}, \frac{12}{13}\right\}$, $>\alpha^{\prime}=\left\{\frac{1}{4}, \frac{3}{4}\right\},>\alpha_{0}^{\prime}=\left\{\frac{1}{12}, \frac{11}{12}\right\},>\alpha_{2}^{\prime}=\left\{\frac{5}{12}, \frac{7}{12}\right\}$, $>\alpha_{01}^{\prime}=\left\{\frac{11}{36}, \frac{25}{36}\right\},>\alpha_{00}^{\prime}=\left\{\frac{1}{36}, \frac{35}{36}\right\},>\alpha_{02}^{\prime}=\left\{\frac{13}{36}, \frac{23}{36}\right\}$.

We now construct a Thurston obstruction $\left\{\delta_{1}, \delta_{2}\right\}$ for $F=g \Perp P_{1}$, which is made of external rays and some part of the equator $E=\left\{\left(\infty \cdot e^{2 \pi i s}, g\right) \mid s \in\right.$ $\mathbf{T}=\mathbb{R} / \mathbb{Z}\}$.
For $\left.t^{\prime}, t \in\right] 0,1[$, set

$E\left[t^{\prime}, t\right]=\left\{\left(\infty \cdot e^{2 \pi i s}, g\right) \mid s \in\left[\min \left\{t^{\prime}, t\right\}, \max \left\{t^{\prime}, t\right\}\right]\right\}$.

For $i=g, P$, if two external rays $R_{i}(\theta), R_{i}\left(\theta^{\prime}\right)$ land at a common point $u \in K_{i}$, set

$$
R_{i}\left(u ; \theta, \theta^{\prime}\right)=R_{i}(\theta) \cup R_{i}\left(\theta^{\prime}\right) .
$$

Now we define

$\delta_{1}=E\left[\frac{1}{8}, \frac{1}{4}\right] \cup R_{P}\left(\alpha^{\prime} ;-\frac{1}{4},-\frac{3}{4}\right) \cup E\left[\frac{3}{4}, \frac{3}{8}\right] \cup R_{g}\left(\alpha ; \frac{3}{8}, \frac{1}{8}\right)$.

$\delta_{1}$ is clearly a nonperipheral simple closed curve in $S_{g, P_{1}}^{2}-P_{F}$. We will see that:

(i) $F^{-1}\left(\delta_{1}\right)$ contains two components $\delta_{2}$ and $\delta_{3}$ with $\operatorname{deg}\left(F: \delta_{2} \rightarrow \delta_{1}\right)=1, \operatorname{deg}\left(F: \delta_{3} \rightarrow \delta_{1}\right)=2$. The curve $\delta_{2}$ is nonperipheral, non-isotopic to $\delta_{1}$ and disjoint with $\delta_{1}$. The curve $\delta_{3}$ is isotopic to $\delta_{1}$ rel $P_{F}$.

(ii) $F^{-1}\left(\delta_{2}\right)$ contains two components $\delta_{4}$ and $\delta_{5}$ with $\operatorname{deg}\left(F: \delta_{4} \rightarrow \delta_{2}\right)=1, \operatorname{deg}\left(F: \delta_{5} \rightarrow \delta_{2}\right)=2 . \delta_{4}$ is peripheral and $\delta_{5}$ is isotopic to $\delta_{1}$ rel $P_{F}$.

Proof. An easy calculation shows that

$$
\begin{aligned}
\delta_{2}= & E\left[\frac{1}{24}, \frac{1}{12}\right] \cup R_{P}\left(\alpha_{0}^{\prime} ;-\frac{1}{12},-\frac{11}{12}\right) \cup \\
& E\left[\frac{11}{12}, \frac{19}{24}\right] \cup R_{g}\left(\alpha_{0} ; \frac{19}{24}, \frac{1}{24}\right), \\
\delta_{3}= & E\left[\frac{3}{8}, \frac{5}{12}\right] \cup R_{P}\left(\alpha_{2}^{\prime} ;-\frac{5}{12},-\frac{7}{12}\right) \cup \\
& E\left[\frac{7}{12}, \frac{11}{24}\right] \cup R_{g}\left(\alpha_{2} ; \frac{11}{24}, \frac{17}{24}\right) \cup \\
& E\left[\frac{17}{24}, \frac{3}{4}\right] \cup R_{P}\left(\alpha^{\prime} ;-\frac{3}{4},-\frac{1}{4}\right) \cup \\
& E\left[\frac{1}{4}, \frac{1}{8}\right] \cup R_{g}\left(\alpha ; \frac{1}{8}, \frac{3}{8}\right), \\
\delta_{4}= & E\left[\frac{1}{72}, \frac{1}{36}\right] \cup R_{P}\left(\alpha_{00}^{\prime} ;-\frac{1}{36},-\frac{35}{36}\right) \cup \\
& E\left[\frac{35}{36}, \frac{67}{72}\right] \cup R_{g}\left(\alpha_{00} ; \frac{67}{72}, \frac{1}{72}\right), \\
\delta_{5}= & E\left[\frac{25}{72}, \frac{13}{36}\right] \cup R_{P}\left(\alpha_{02}^{\prime} ;-\frac{13}{36},-\frac{23}{36}\right) \cup \\
& E\left[\frac{23}{36}, \frac{43}{72}\right] \cup R_{g}\left(\alpha_{02} ; \frac{43}{72}, \frac{49}{72}\right) \cup \\
& E\left[\frac{49}{72}, \frac{25}{36}\right] \cup R_{P}\left(\alpha_{01}^{\prime} ;-\frac{25}{36},-\frac{11}{36}\right) \cup \\
& E\left[\frac{11}{36}, \frac{19}{72}\right] \cup R_{g}\left(\alpha_{01} ; \frac{19}{72}, \frac{25}{72}\right) .
\end{aligned}
$$

We get (i) and (ii) from Figure 4.

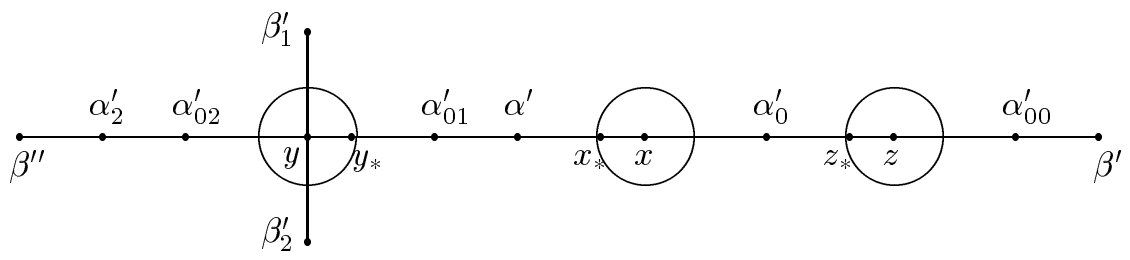

FIGURE 3. Extended Hubbard tree for $P_{1}$. 

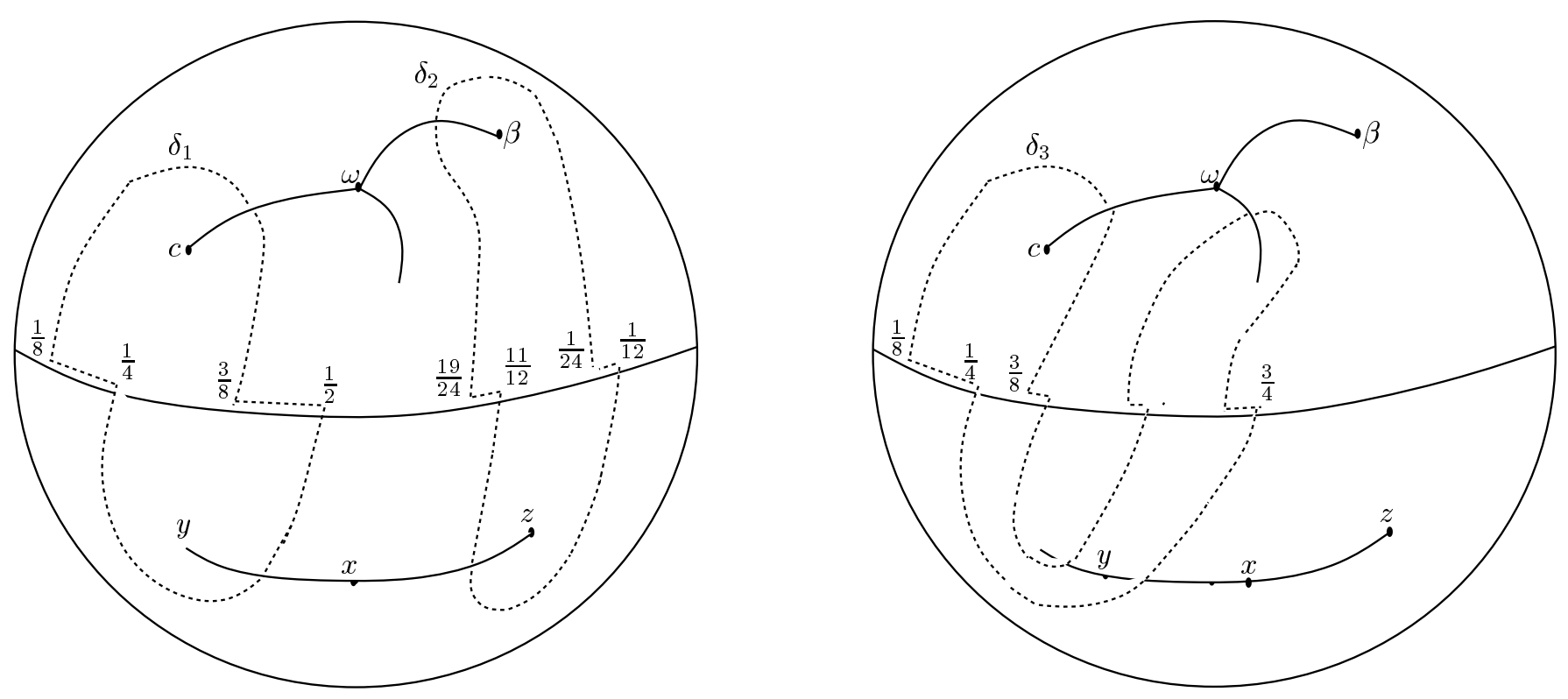

FIGURE 4. The curves $\delta_{1}, \delta_{2}$, and $\delta_{3}$.

Now $\Gamma=\left\{\delta_{1}, \delta_{2}\right\}$ is a multicurve whose Thurston linear transformation $F_{\Gamma}: \mathbb{R}^{\Gamma} \rightarrow \mathbb{R}^{\Gamma}$ is given, in the basis $\delta_{1}, \delta_{2}$, by the matrix

$$
\left(\begin{array}{cc}
\frac{1}{2} & \frac{1}{2} \\
1 & 0
\end{array}\right)
$$

whose leading eigenvalue $\lambda_{\Gamma}$ equals 1 , with eigenvector $\left(\begin{array}{l}1 \\ 1\end{array}\right)$. Clearly $\Gamma$ is an irreducible obstruction but not a removable Levy cycle. It is also a nested obstruction. By definition, $F$ is not weakly equivalent to a rational map; that is, $g, P_{1}$ are not matable.

\section{GENERAL ANALYSIS ON BRANCHED COVERINGS AND MATINGS}

Section 3A studies the ray-equivalence of a mating. Sections 3B-3H contain useful techniques to deal with Thurston obstructions, intersection problems of a multicurve with a Hubbard tree, weak equivalence to a rational map, etc. We will see some applications in the next section.

\section{A. The Quotient is a Sphere}

A mating $F=f \Perp g$ posesses a ray-equivalence relation, which gives more detailed information about Thurston obstructions and Levy cycles, such as results in Theorem 1.4 and Theorem 1.5.

In this section we want to analyze some properties of the ray-equivalence relation of a mating, and give some conditions for $S^{2} / \sim_{\text {ray }}$ to be a topological 2 sphere.

First a general result about a Hubbard tree; for a proof, see [Tan 1997].

Proposition 3.1. For a postcritically finite polynomial $f$, every point $x \in J_{f}$ with more than one external ray falls eventually into the Hubbard tree of $f$.

We say that an equivalence relation $\sim$ in a compact metric space $X$ is closed if the graph of $\sim$ in $X \times X$ is a closed set. This is equivalent to say that any sequences $x_{n} \rightarrow x, y_{n} \rightarrow y$ such that $x_{n} \sim y_{n}$ for each $n$ satisfy $x \sim y$.

Proposition 3.2. Suppose $f, g$ are degree-d monic polynomials with locally connected Julia set. Set $F=$ $f \Perp g$. If there is $K<\infty$ such that $\#[x] \cap E \leq K$ for every $x \in S_{f, g}^{2}$, ray-equivalence is closed.

Proof. An external ray $R(\theta)=R_{f}(\theta) \cup R_{g}(-\theta) \subset S_{f, g}^{2}$ is a connected arc. Since the Julia set of both $f$ and $g$ is locally connected, the rays move continuously. So, as $\theta_{n} \rightarrow \theta_{0}$ as $n \rightarrow \infty$, we have $R\left(\theta_{n}\right) \rightarrow R\left(\theta_{0}\right)$ with respect to the Hausdorff distance on the space of closed subsets of $S_{f, g}^{2}$.

Suppose $x_{n}, y_{n} \in S_{f, g}^{2}$ such that $x_{n} \sim_{\text {ray }} y_{n}$ for all $n$, and $x_{n} \rightarrow x_{0}, y_{n} \rightarrow y_{0}$. We need to prove $x_{0} \sim_{\text {ray }} y_{0}$. 
For each $n$, there is a collection of angles $\theta_{n, 1}, \theta_{n, 2}$, $\ldots, \theta_{n, k_{n}}$ such that

$x_{n} \in R\left(\theta_{n, 1}\right), y_{n} \in R\left(\theta_{n, k_{n}}\right), R\left(\theta_{n, i}\right) \cap R\left(\theta_{n, i+1}\right) \neq \varnothing$

for $i=1,2, \ldots, k_{n}-1$.

By assumption, we have $k_{n} \leq K$ for each $n$. Taking a subsequence if necessary, we may assume that $k_{n}$ is a constant $k$ for all $n$. By taking again subsequences, we may assume that $\theta_{n, i} \rightarrow \theta_{i}($ as $n \rightarrow \infty)$ for some $\theta_{i} \in \mathbf{T}$ and for each $i=1,2, \ldots, k$.

By the continuity of external rays $R(\theta)$, we have

$$
R\left(\theta_{n, i}\right) \rightarrow R\left(\theta_{i}\right), \text { as } n \rightarrow \infty,
$$

for $i=1,2, \ldots, k$. It follows that $x_{0} \in R\left(\theta_{1}\right), y_{0} \in$ $R\left(\theta_{k}\right)$, and $R\left(\theta_{i}\right) \cap R\left(\theta_{i+1}\right) \neq \varnothing$ for $i=1,2, \ldots, k-1$. Hence

$$
\bigcup_{i=1}^{k} R\left(\theta_{i}\right)
$$

is connected, and therefore $x_{0} \sim_{\text {ray }} y_{0}$.

Corollary 3.3. If, moreover, no equivalence class of $\sim$ separates $S_{f, g}^{2}$, then $S_{f, g}^{2} / \sim$ is homeomorphic to $S^{2}$.

Proof. This is a simple application of the Moore's theorem [1925]: If $G$ is a partition of $S^{2}$ into compact, connected, nonseparating sets that gives rise to a closed equivalent relation, the quotient $S^{2} / G$ is homeomorphic to $S^{2}$.

Proposition 3.4. The induced quotient map $[f \Perp g]$ from $S_{f, g}^{2} / \sim$ to itself is again a branched covering.

Proof. Denote by $V$ the union of the finitely many ray classes containing critical values of $f$. Then $f^{-1}(V)$ consists again of finitely many ray classes and $f$ : $S^{2}-f^{-1}(V) \rightarrow S^{2}-V$ is an unbranched covering. Let $y \in \pi\left(S^{2}-V\right)$ and $Y$ a simply connected open neighborhood of $y$. Since $\pi: S^{2}-V \rightarrow \pi\left(S^{2}-V\right)$ is proper, $\pi^{-1}(Y)$ is again simply connected [Douady and Douady 1979]. Thus each connected component of $f^{-1} \pi^{-1}(Y)$ is simply connected. So is each connected component of $\pi f^{-1} \pi^{-1}(Y)$. This proves that $[f \Perp g]$ is a covering of $S^{2}$ minus finitely many points. It extends then naturally to a branched covering of $S^{2}$.

\section{B. Irreducible Obstructions}

Let $F: S^{2} \rightarrow S^{2}$ be a postcritically finite branched covering. Recall that, for a multicurve

$$
\Gamma=\left\{\gamma_{1}, \ldots, \gamma_{n}\right\}
$$

the corresponding matrix $F_{\Gamma}=\left(a_{i j}\right)$ is defined by

$$
a_{i j}=\sum_{\gamma^{\prime} \in F^{-1}\left(\gamma_{j}\right), \gamma^{\prime} \sim \gamma_{i}} \frac{1}{\operatorname{deg}\left(F: \gamma^{\prime} \rightarrow \gamma_{j}\right)} .
$$

See [Gantmacher 1959] for the definition and properties of irreducible nonnegative matrices.

Definition. A multicurve $\Gamma$ is called irreducible if $F_{\Gamma}$ is irreducible. $\Gamma$ is called an irreducible obstruction if $F_{\Gamma}$ is irreducible and $\lambda\left(F_{\Gamma}\right) \geq 1$. In other words, for any $(i, j) \in\{1, \ldots, k\}^{2}, k=\# \Gamma$, there is an integer $n$, a component $\gamma^{\prime}$ of $F^{-n}\left(\gamma_{j}\right)$ isotopic to $\gamma_{i}$, and for $1 \leq m \leq n, F^{m}\left(\gamma^{\prime}\right)$ is isotopic to a curve in $\Gamma$. Levy cycles are examples of irreducible obstructions. A multicurve $\Gamma$ is $F$-invariant, if for any $\gamma \in \Gamma$, each connected component of $F^{-1}(\gamma)$ is either peripheral or isotopic to a curve in $\Gamma$ rel $P_{F}$.

Lemma 3.5. Any Thurston obstruction contains an irreducible obstruction. Any irreducible obstruction is isotopically contained in a F-invariant Thurston obstruction. As a consequence, in the case that $F$ has a hyperbolic orbifold, $F$ is not equivalent to a rational map if and only if $F$ has an irreducible obstruction.

Proof. Let $\Gamma$ be a Thurston obstruction for $F$. Then $\Gamma$ can be considered as disjoint union of submulticurves $\Gamma_{m}$ such that $F_{\Gamma_{m}}$ is irreducible. Moreover, $\lambda\left(F_{\Gamma}\right)=\max _{m}\left\{\lambda\left(F_{\Gamma_{m}}\right)\right\}$. Any $\Gamma_{m}$ with $\lambda\left(F_{\Gamma_{m}}\right) \geq 1$ is an irreducible obstruction.

Now let $\Gamma_{0}$ be an irreducible obstruction for $F$. Let $\Gamma_{1}$ be the set of isotopy classes of curves in $F^{-1}\left(\Gamma_{0}\right)$. Then $\Gamma_{0} \subset \Gamma_{1}$ (isotopically). To show that $\Gamma_{1}$ is again a multicurve we should check that two classes in $\Gamma_{1}$ do not intersect (isotopically). Since curves in $\Gamma_{0}$ are disjoint from each other, so are curves in $F^{-1}\left(\Gamma_{0}\right)$, so are isotopy classes in $\Gamma_{1}$. Now we can take inductively $\Gamma_{n}$ to be the set of isotopy classes of $F^{-1}\left(\Gamma_{n-1}\right)$. Then $\Gamma_{n}$ is a multicurve containing $\Gamma_{n-1}$. There is a $n$ such that $\Gamma_{n+1}=\Gamma_{n}$, i.e. $\Gamma_{n}$ is an $F$-invariant multicurve. It remains to show $\lambda\left(F_{\Gamma_{n}}\right) \geq 1$. Let $F_{\Gamma_{n}}=\left(a_{i j}\right)_{m \times m}, F_{\Gamma_{0}}=\left(b_{i j}\right)_{k \times k}$. We have $k \leq m$. Number curves in $\Gamma_{n}$ so that the 
first $k$ ones are in $\Gamma_{0}$. Define a new matrix $\left(c_{i j}\right)_{m \times m}$ as follows: $c_{i j}=b_{i j}$ if $i, j \leq k$, and $c_{i j}=0$ elsewhere. Then $a_{i j} \geq c_{i j}$ for each $(i, j)$. By PerronFrobenius theory on nonnegative matrices, we have $\lambda\left(\left(a_{i j}\right)\right) \geq \lambda\left(\left(c_{i j}\right)\right)=\lambda\left(\left(b_{i j}\right)\right)=\lambda\left(F_{\Gamma_{0}}\right) \geq 1$. So $\Gamma_{n}$ is an $F$-invariant Thurston obstruction.

\section{C. Disc-Components in the Complement}

Let $F$ be a postcritically finite branched covering. Let $A, B$ be two subsets of $S^{2}$. We say that $A$ is isotopically contained in $B$ if there is a homeomorphism $\psi$ of $S^{2}$, isotopic to the identity rel $P_{F}$, such that $\psi(A) \subset B$. If there is such a $\psi$ with $\psi(A)=B$, we say that $A$ is isotopic to $B$.

Lemma 3.6. Let $\Gamma$ be an irreducible multicurve for $F$ (for example, a Levy cycle). Then

(a) Each connected component of $S^{2}-F^{-1}(\Gamma)$ is exactly a connected component of $F^{-1}(A)$, where $A$ is some connected component of $S^{2}-\Gamma$.

(b) Any connected component of $S^{2}-F^{-1}(\Gamma)$ is isotopically contained in a connected component of $S^{2}-\Gamma$.

(c) Let $D^{\prime}$ be a disc-component of $F^{-1}(D)$ for $D$ a component of $S^{2}-\Gamma$, such that $\partial D^{\prime}$ is isotopic to a curve $\gamma_{i}$ in $\Gamma$, then $D^{\prime}$ is isotopic to a disccomponent of $S^{2}-\Gamma$ with boundary $\gamma_{i}$. And if the components of $F^{-1}(D)$ are discs, at least one of them must be isotopic to a disc-component of $S^{2}-\Gamma$.

Proof. Statement (a) is due to general properties of a branched covering.

For (b), since any curve in $\Gamma$ is isotopic to a curve in $F^{-1}(\Gamma)$, there is a homeomorphism $\psi$ isotopic to the identity rel $P_{F}$ mapping $\Gamma$ into $F^{-1}(\Gamma)$. So $\psi^{-1}$ maps a component of $S^{2}-F^{-1}(\Gamma)$ into a component of $S^{2}-\Gamma$.

For (c), suppose that $D^{\prime}$ is isotopically contained in $A$ and that $A$ is not a disc-component of $S^{2}-\Gamma$. Since each boundary curve of $A$ is nonperipheral, $\partial D^{\prime}$ can not be isotopic to any of them. So $A$ is a disc, $\partial D^{\prime}$ is isotopic to $\partial A$ and $D^{\prime}$ is isotopic to $A$. Assume now that the components of $F^{-1}(D)$ are discs. Since at least one curve $\gamma^{\prime}$ of $F^{-1}(\partial D)$ is isotopic to a curve in $\Gamma$, the unique disc-component $D^{\prime}$ of $S^{2}-F^{-1}(\Gamma)$ bounded by $\gamma^{\prime}$ is isotopic to a disc-component of $F$.
The following result combines work of S. Levy, M. Rees, the authors and others.

Proposition 3.7. $\Gamma$ is a removable Levy cycle if and only if $\Gamma$ is an irreducible obstruction and there is a disc-component $D$ of $S^{2}-\Gamma$, such that for all $n$, the connected components of $F^{-n}(D)$ are discs. In this case $F^{-1}(\Gamma)$ contains a unique subset $\Gamma^{\prime}$ isotopic to $\Gamma$ such that $F: \Gamma^{\prime} \rightarrow \Gamma$ is a homeomorphism. If $\Gamma$ is an irreducible obstruction and each disc component of $S^{2}-\Gamma$ contains at most one critical value, then $\Gamma$ is a removable Levy cycle.

Proof. Necessity is a consequence of the definition. To prove sufficiency, set $\gamma=\partial D$. Given any $\gamma_{j} \in \Gamma$, by irreducibility, there is an integer $n$ such that a curve $\gamma^{\prime}$ in $F^{-n}(\gamma)$ is isotopic to $\gamma_{j}$. Since $\gamma^{\prime}$ bounds a disc-component $D^{\prime}$ of $S^{2}-F^{-n}(\Gamma)$, by the above lemma, $D^{\prime}$ is isotopic to a disc-component $D_{j}$ of $S^{2}-\Gamma$ bounded by $\gamma_{j}$. Thus $S^{2}-\Gamma$ has at most one non-disc-component.

For simplicity, assume that $\# \Gamma=k>1$ (the case $\# \Gamma=1$ is left to the reader). Then $S^{2}-\Gamma$ has exactly $k$ disc-components $D_{1}, \ldots, D_{k}$, with $\gamma_{i}=$ $\partial D_{i}$. We claim that $F_{\Gamma}=\left(b_{i j}\right)$, with

$$
b_{i j}=\sum_{\substack{D^{\prime} \text { isotopic to } D_{i}, F\left(D^{\prime}\right)=D_{j}}} \frac{1}{\operatorname{deg}\left(F: D^{\prime} \rightarrow D_{j}\right)} .
$$

Let $\gamma^{\prime}$ be a component of $F^{-1}\left(\gamma_{j}\right)$. It bounds a unique disc-component $D^{\prime}$ of $S^{2}-F^{-1}(\Gamma)$ such that $F\left(D^{\prime}\right)=D_{j}$. Moreover $\operatorname{deg}\left(F: \gamma^{\prime} \rightarrow \gamma_{j}\right)=\operatorname{deg}(F:$ $\left.D^{\prime} \rightarrow D_{j}\right)$. By the above lemma, $\gamma^{\prime}$ is isotopic to $\gamma_{i}$ if and only if $D^{\prime}$ is isotopic to $D_{i}$. By definition of $F_{\Gamma}$, we get the claim.

Now for each $j$, there is at most one $D^{\prime}$ in $F^{-1}\left(D_{j}\right)$ isotopic to $D_{i}$. So the sum in $b_{i j}$ contains at most one term. On the other hand, for each $i$, there is at most one $j$ such that $b_{i j} \neq 0$, because $F^{-1}\left(D_{j}\right)$ and $F^{-1}\left(D_{j^{\prime}}\right)$ are isotopically disjoint whenever $j \neq$ $j^{\prime}$. By the irreducibility and the fact $\lambda(\Gamma) \geq 1$, we conclude that, after a suitable numeration, $b_{i(i+1)}=$ $1, b_{k 1}=1$ and $b_{i j}=0$ elsewhere. This proves that $F^{-1}(\Gamma)$ contains a unique subset $\Gamma^{\prime}$ satisfying the properties in the proposition and $\Gamma$ is a degenerate Levy cycle. Since the components of $F^{-n}\left(D_{1}\right)$ are discs for all $n, \Gamma$ is also removable.

In the last case of the proposition, take $D$ a disccomponent. Since it contains at most one critical 
value, all components of $F^{-1}(D)$ are discs, each is isotopically contained in a component of $S^{2}-\Gamma$, therefore contains at most one critical value. By induction, all components of $F^{-n}(D)$ are discs.

From this we get again a result of M. Rees and a result of S. Levy:

Corollary 3.8. Any irreducible obstruction of a quadratic branched covering contains a Levy cycle.

Proof. Let $\Gamma$ be an irreducible obstruction for $F$.

A quadratic map $F$ has exactly two critical values.

If some curve $\gamma \in \Gamma$ separates the two critical values of $F$, then we are in the case to apply the above proposition. So $\Gamma$ must be a removable Levy cycle (by looking at the degree, we can even prove that $\lambda(\Gamma)<1$, a contradiction. So in fact this situation does not occur). If the two critical values of $F$ are contained in the same component of $S^{2}-\Gamma$, then $F^{-1}(\gamma)$ for each $\gamma \in \Gamma$ consists of two curves and each of them is mapped by $F$ to $\gamma$ with degree one. This implies every periodic cycle in $\Gamma$ is a Levy cycle.

\section{D. Geometric Intersection Number}

Let $f: S^{2} \rightarrow S^{2}$ be a postcritically finite branched covering. We say that $\eta$ is a nontrivial open arc in $S^{2}-P_{f}$ if $\eta=h(] 0,1[)$, where $h:[0,1] \rightarrow S^{2}$ is a continuous mapping, injective on $] 0,1[$, with $h(0), h(1) \in P_{f}$ and $h(] 0,1[) \cap P_{f}=\varnothing$, moreover $h$ is not homotopic to a constant map relative to its boundary values. Denote by $[\eta]$ the isotopy class $\left(\right.$ rel $\left.P_{f}\right)$ of $\eta$.

Denote by $\mathcal{L}$ the set of isotopy classes of nonperipheral simple closed curves and nontrivial open $\operatorname{arcs}$ in $S^{2}-P_{f}$, and by $\mathcal{R}$ the real linear space generated by $\mathcal{L}$. For $[\eta],[\zeta] \in \mathcal{L}$, we define the geometric intersection number by:

$$
[\eta] \cdot[\zeta]=\inf \left\{\# \eta^{\prime} \cap \zeta^{\prime} \mid \eta^{\prime} \in[\eta], \zeta^{\prime} \in[\zeta]\right\} .
$$

Note that $[\eta] \cdot[\eta]=0$. This geometric intersection number can be extended bilinearly to $\mathcal{R} \times \mathcal{R}$.

We define a linear transformation $f_{\#}: \mathcal{R} \rightarrow \mathcal{R}$ by

$$
f_{\#}([\eta])=\sum_{\eta^{\prime} \subset f^{-1}(\eta)}\left[\eta^{\prime}\right]
$$

where the sum is taken over all connected components of $f^{-1}(\eta)$, and again $\left[\eta^{\prime}\right]$ denotes the isotopy class of $\eta^{\prime}$, which is zero if $\eta^{\prime}$ is a) a peripheral closed curve, b) an open arc with at least one end point out of $P_{f}$, or c) an open arc isotopic to a point $\left(\right.$ rel $\left.P_{f}\right)$. It is clear that $f_{\#}([\eta])$ does not depend on the choice of the representative of $[\eta]$, and $\left(f_{\#}\right)^{n}=\left(f^{n}\right)_{\#}$.

Assume that a finite subset $\left\{\left[\gamma_{1}\right], \ldots,\left[\gamma_{k}\right]\right\}$ of $\mathcal{L}$ admits a representative $\Gamma=\left\{\gamma_{1}, \ldots, \gamma_{k}\right\}$ such that $\gamma_{i} \cap \gamma_{j}=\varnothing$ for $i \neq j$. We define two linear transformations $f_{\Gamma}: \mathbb{R}^{\Gamma} \rightarrow \mathbb{R}^{\Gamma}$ and $f_{\#, \Gamma}: \mathbb{R}^{\Gamma} \rightarrow \mathbb{R}^{\Gamma}$ by

$$
\begin{aligned}
f_{\Gamma}(\gamma) & =\sum_{\gamma^{\prime} \subset f^{-1}(\gamma)} \frac{1}{\operatorname{deg}\left(f: \gamma^{\prime} \rightarrow \gamma\right)}\left[\gamma^{\prime}\right]_{\Gamma}, \\
f_{\#, \Gamma}(\gamma) & =\sum_{\gamma^{\prime} \subset f^{-1}(\gamma)}\left[\gamma^{\prime}\right]_{\Gamma}
\end{aligned}
$$

for every $\gamma \in \Gamma$, where $\left[\gamma^{\prime}\right]_{\Gamma}$ denotes the element in $\Gamma$ isotopic to $\gamma^{\prime}$ if it exists and 0 otherwise. Note that $\operatorname{deg}\left(f: \eta^{\prime} \rightarrow \eta\right)=1$ whenever $\eta$ is an arc. We have $\left(f_{\#, \Gamma}\right)^{n} \leq\left(f^{n}\right)_{\#, \Gamma}$, and $\left(f_{\Gamma}\right)^{n}(\gamma) \leq\left(f^{n}\right)_{\Gamma}(\gamma)$, since some $n$-th preimage $\gamma^{\prime}$ of $\gamma$ might come back to $\Gamma$ but $f\left(\gamma^{\prime}\right) \notin \Gamma$.

For $\Gamma$ as above, set $\tilde{\Gamma}\left(f^{n}\right)$ to be the union of those components of $f^{-n}(\Gamma)$ that are isotopic to elements of $\Gamma$ (the case $n=1$ being written simply $\tilde{\Gamma}$ ). If $\Gamma$ is irreducible, each component of $\Gamma$ is isotopic to some (not necessarily unique) component of $\tilde{\Gamma}\left(f^{n}\right)$.

A different form of the next theorem appeared in the preprint version of this paper. The generalization given here is proved in [Pilgrim and Tan 1998].

Theorem 3.9. Let $f: S^{2} \rightarrow S^{2}$ be a postcritically finite branched covering. Assume that two finite subsets of $\mathcal{L}$ admit representatives $\Gamma=\left\{\gamma_{1}, \ldots, \gamma_{k}\right\}$ and $\Lambda=$ $\left\{\lambda_{1}, \ldots, \lambda_{l}\right\}$ such that $\gamma_{i} \cap \gamma_{j}=\lambda_{i} \cap \lambda_{j}=\varnothing$ for $i \neq j$. Assume furthermore that

(0) $\#(\Gamma \cap \Lambda)=\Gamma \cdot \Lambda$;

(1) $f_{\Gamma}$ has neither zero row nor zero column, with leading eigenvalue at least 1 ;

(2) $\tilde{\Lambda}\left(\subset f^{-1}(\Lambda)\right)$ has a subset $\tilde{\Lambda}^{\prime}$ isotopic to $\Lambda$ and $f: \tilde{\Lambda}^{\prime} \rightarrow \Lambda$ is a homeomorphism.

Then either

1. $\Gamma \cdot \Lambda=0$ and $\Gamma \cdot f^{-n}(\Lambda)=0, f^{-n}(\Gamma) \cdot \Lambda=0$ for all $n \geq 1$; or

2. $\Gamma \cdot \Lambda \neq 0$ and

a. $\tilde{\Gamma}$ is isotopic to $\Gamma, \tilde{\Lambda}$ is isotopic to $\Lambda$, the mappings $f: \tilde{\Gamma} \rightarrow \Gamma$ and $f: \tilde{\Lambda} \rightarrow \Lambda$ are homeomorphisms (so $\left.\left.\tilde{\Lambda}^{\prime}=\tilde{\Lambda}\right), \tilde{\Gamma} \cap\left(f^{-1}(\Lambda)-\tilde{\Lambda}\right)\right)=\varnothing$ and $\tilde{\Lambda} \cap\left(f^{-1}(\Gamma)-\tilde{\Gamma}\right)=\varnothing$. More precisely, each 
component of $\Gamma$ is isotopic to a unique component of $\tilde{\Gamma}$, each component of $\Lambda$ is isotopic to a unique component of $\tilde{\Lambda}$, and

i. for each $\gamma \in \Gamma$, there is exactly one component $\gamma^{\prime} \subset f^{-1}(\gamma)$ such that $\gamma^{\prime} \cap \tilde{\Lambda} \neq \varnothing$, moreover, $\gamma^{\prime}$ is the unique component of $f^{-1}(\gamma)$ isotopic to an element of $\Gamma$;

ii. for each $\lambda \in \Lambda$, there is exactly one component $\lambda^{\prime}$ of $f^{-1}(\lambda)$ such that $\lambda^{\prime} \cap \tilde{\Gamma} \neq \varnothing$, moreover, $\lambda^{\prime}$ is the unique component of $f^{-1}(\lambda)$ isotopic to an element of $\Lambda$.

b. The transformations $f_{\#, \Gamma}$ and $f_{\#, \Lambda}$ are transitive permutations of the basis vectors.

c. The above results remain true if we replace $f$ by $f^{n}$, for any $n \geq 1$ (though transitivity may fail).

d. For any $\lambda$ isotopic to a component of $f^{-n}(\Lambda)$ for some $n>1$ but not isotopic to a component of $\Lambda, \Gamma \cdot \lambda=0$. Similarly, for any $\gamma$ isotopic to a component of $f^{-n}(\Gamma)$ for some $n>1$ but not isotopic to a component of $\Gamma$, $\Lambda \cdot \gamma=0$.

Corollary 3.10. If $\Lambda$ is a Levy cycle and $\Gamma$ an irreducible obstruction with $\Gamma \cdot \Lambda \neq 0$, then $\Gamma$ is again a Levy cycle.

Corollary 3.11. Suppose that in a mating $f \Perp g$, one polynomial, say $g$, has a "star-like" Hubbard tree $H_{g}$, that is, $H_{g}$ has only one branch point $\alpha$ and $g: H_{g} \rightarrow H_{g}$ is a homeomorphism. Then any irreducible obstruction of $f \Perp g$ is a Levy cycle, whose limit set (according to Theorem 1.4) coincides with the ray class $[\alpha]$. Moreover, if the rays of $\alpha$ form a single orbit by $g$ then either there is $\theta$ such that $R_{f}(\theta) \cup R_{g}(-\theta)$ links $\alpha$ to a fixed point of $f$, or $[\alpha]$ is a tree.

Proof. Consider $H_{g}$ as a periodic cycle of isotopy classes of arcs. For $\Gamma$ an irreducible obstruction of $f \Perp g$, we must have $\Gamma \cdot H_{g} \neq 0$ (otherwise $\Gamma$ reduces to an obstruction for $f$ ). So by the above theorem $\Gamma$ is a Levy cycle, with limit set passing through $H_{g}$. The only possibility is $[\alpha]$, since $\alpha$ is the unique point of $H_{g}$ in the Julia set.

Denote by $d$ the degree of $f$ and $g$. By assumption, the map $\theta \mapsto d \theta$ acts as a cyclic permutation on the set $-\theta_{1}, \ldots,-\theta_{k}$ of external angles of $\alpha$. Denote by $\alpha_{i}$ the landing point of $R_{f}\left(\theta_{i}\right)$. If $\alpha_{i}=\alpha_{j}$ for some $i \neq j$, then by the cyclic permutation property and the fact that $F$ preserves the cyclic order at branch points of $[\alpha]$, we have $\alpha_{1}=\alpha_{2}=\cdots=\alpha_{k}$ and it is a fixed point of $f$. Assume now the $\alpha_{i}$ 's are pairwise distinct. Fix an index $j$ and $\eta \neq \theta_{j}$ (if any) an external angle of $\alpha_{j}$. Then the ray $R_{f}(\eta) \cup R_{g}(-\eta)$ lands at a periodic point $u \in J_{g}-H_{g}$ (since $H_{g} \cap J_{g}=\{\alpha\}$ ). The point $u$ has no other external rays (Proposition 3.1). So $[\alpha]$ is a tree.

\section{E. Removing Levy Cycles}

Let $F: S^{2} \rightarrow S^{2}$ be a postcritically finite branched covering. We describe here how to modify $F$ in order to delete simultaneously a maximal number of removable Levy cycles.

Recall that each removable Levy cycle $\Gamma$ with $n$ curves decomposes the sphere into $n$ disc-components $B_{1}, \ldots, B_{n}$ and one extra component $C$ (which is not a disc unless $n=1)$, such that each $F^{-1}\left(B_{j}\right)$ has a component $B_{j}^{\prime}$ isotopic to $B_{j-1}(\bmod n)$, with $\operatorname{deg}\left(F: B_{j}^{\prime} \rightarrow B_{j}\right)=1$. Moreover for all $k>0$, all components of $F^{-k}\left(B_{1}\right)$ are discs. Therefore for all $k>0$ and $j=1, \ldots, n$, all components of $F^{-k}\left(B_{j}\right)$ are discs. Set $\mathcal{B}(\Gamma)=\bigcup_{j} B_{j}$.

Denote by $\Sigma=\Sigma(F)$ the set of isotopy classes of removable Levy cycles for $F$. We define a partial order on $\Sigma$ : we say that $\xi_{1}<\xi_{2}$ if there are representatives $\Gamma_{1}, \Gamma_{2}$ of $\xi_{1}, \xi_{2}$ such that $\mathcal{B}\left(\Gamma_{1}\right) \subset \mathcal{B}\left(\Gamma_{2}\right)$.

If the geometric intersection number $\xi_{1} \cdot \xi_{2}$ is zero for two elements of $\Sigma$, either one is smaller than the other, or there are representatives $\Gamma_{1}, \Gamma_{2}$ of $\xi_{1}, \xi_{2}$ such that $S^{2}-\Gamma_{1} \cup \Gamma_{2}$ has exactly one non-disccomponent, and $\# \Gamma_{1}+\# \Gamma_{2}$ disc-components.

Denote by $\Sigma^{\prime}=\Sigma^{\prime}(F)$ the set of $\xi \in \Sigma$, satisfying that $\xi$ is maximal in $\Sigma$ and $\xi \cdot \Gamma=0$ for any Levy cycle $\Gamma$ (see Sections $3 \mathrm{~F}-3 \mathrm{H}$ for examples and counterexamples).

Denote by $\mathcal{G}_{d}$ the set of equivalence classes of postcritically finite branched coverings of degree $d$.

Theorem 3.12. There is a mapping $s: \mathcal{G}_{d} \rightarrow \mathcal{G}_{d}$ with these properties:

(a) it fixes every class which contains a rational map;

(b) if $s([F])=[G]$, there is a bijection between the two sets $A_{F}=\{$ isotopy classes of irreducible obstructions of $F$ not smaller than or equal to an 
element of $\left.\Sigma^{\prime}(F)\right\}$ and $A_{G}=\{$ isotopy classes of irreducible obstructions of $G\}$;

(c) $\Sigma^{\prime}(G)=\varnothing$ for $[G] \in s\left(\mathcal{G}_{d}\right)$ and $s \circ s=s$.

Proof. For any $\xi \in \Sigma^{\prime}(F)$, we have $\xi \cdot \Gamma=0$ for any irreducible obstruction $\Gamma$, since by Corollary 3.10, any $\Gamma$ with $\xi \cdot \Gamma \neq 0$ is a Levy cycle.

I. Construction of s. We define $s([F])=[F]$ if $\Sigma^{\prime}(F)=$ $\varnothing$. Assume that $\Sigma^{\prime}=\Sigma^{\prime}(F) \neq \varnothing$.

For any $\xi \in \Sigma^{\prime}$, the set $\xi \cap P_{F}$ is defined to be $\mathcal{B}(\Gamma) \cap P_{F}$, for any $\Gamma$ a representative of $\xi$. The set $\xi \cap P_{F}$ is not empty, independent of the choice of $\Gamma$, and contains only periodic non-superattracting elements of $P_{F}$. For $\xi, \xi^{\prime} \in \Sigma^{\prime}$ distinct, we have $\left(\xi \cap P_{F}\right) \cap\left(\xi^{\prime} \cap P_{F}\right)=\varnothing$. Since $\# P_{F}$ is finite, the set $\Sigma^{\prime}$ contains only finitely many elements $\xi_{1}, \ldots, \xi_{k}$.

Step 1: The mapping $\mathrm{H}$ and the integer $\mathrm{n}$. For each $i$, choose $\Gamma_{i}$ a representative of $\xi_{i}$ such that $\Gamma_{i}, \Gamma_{j}$ are disjoint for $i \neq j$. Denote by $\Gamma=\bigcup_{i=1}^{k} \Gamma_{i}$, and $\mathcal{B}=\bigcup_{i=1}^{k} \mathcal{B}\left(\Gamma_{i}\right)$. From the proof of Proposition 3.7, the set $\Gamma^{\prime}$ of curves in $F^{-1}(\Gamma)$ homotopic to some curve in $\Gamma$ is isotopic to $\Gamma$, and $F: \Gamma^{\prime} \rightarrow \Gamma$ is a homeomorphism.

Let $\Psi: S^{2} \rightarrow S^{2}$ be a homeomorphism isotopic to the identity rel $P_{F}$, such that $\Psi(\Gamma)=\Gamma^{\prime}$. Set $H=F \circ \Psi$. Then $H$ is Thurston equivalent to $F$, $P_{H}=P_{F}, H(\Gamma)=\Gamma$, and $H: \mathcal{B} \rightarrow \mathcal{B}$ is a homeomorphism.

Note that $\Gamma \subset H^{-1}(\Gamma)$ and $\# H^{-1}(\Gamma) \geq 2$. By induction $H^{-(n-1)}(\Gamma) \subset H^{-n}(\Gamma)$. There is a minimal integer $n$ such that

$$
\left(H^{-(n+1)}(\mathcal{B})-H^{-n}(\mathcal{B})\right) \cap\left(P_{H} \cup \Omega_{H}\right)=\varnothing,
$$

where $\Omega_{H}$ is the set of critical points of $H$.

Step 2: Marking points. In each component $B$ of $\mathcal{B}$ we mark a point $y$ such that $y$ has the same period as $B$ (such a point exists according to Brouwer's fixed point theorem), and $H$ maps marked point to marked point. Inductively, for $j=1, \ldots, n$, we mark a point $y$ in each component $B$ of $H^{-j}(\mathcal{B})-$ $H^{-(j-1)}(\mathcal{B})$ such that $H(y)$ is the marked point of $H(B)$.

Step 3: Modifications. We will modify the map in each marked disc $(B, y)$ of $H^{-n}(\mathcal{B})$ containing a critical point (such a disc is surely not periodic, that is, $B \notin \mathcal{B})$. Set $\left(B^{\prime}, y^{\prime}\right)=H((B, y))$. Denoting by
$D$ the unit disk, let $\varphi: \bar{B} \rightarrow \bar{D}$ be an orientationpreserving homeomorphism with $\varphi(y)=0$, and likewise $\varphi^{\prime}: \overline{B^{\prime}} \rightarrow \bar{D}$ with $\varphi^{\prime}\left(y^{\prime}\right)=0$. Extend the boundary mapping $g=\varphi^{\prime} \circ H_{\partial B} \circ \varphi^{-1}$ to a mapping (denoted again by $g$ ) of the whole disk by $t \cdot w \mapsto$ $t \cdot g(w), 0 \leq t \leq 1$. It is a covering branched only at 0 , and its degree coincides with $\operatorname{deg}\left(H: \partial B \rightarrow \partial B^{\prime}\right)$.

Now we define $G: S^{2} \rightarrow S^{2}$ to be: on each disc $B$ of $H^{-n}(\mathcal{B})$ containing a critical point, set $G_{B}=$ $\left(\varphi^{\prime}\right)^{-1} \circ g \circ \varphi$. Elsewhere set $G=H$.

Step 4: Independence of [G] on the choices in the construction. At first another choice of $\Gamma$ in its isotopy class and $\Psi$ will give always a mapping $H$ in the class of $F$. The integer $n$ in Step 1 remains unchanged. Next another choice of the marked orbit and the maps $\varphi, \varphi^{\prime}$ will give a different extension of $\left.H\right|_{\partial B}$ for $B$ a component of $H^{-n}(\mathcal{B})$ containing a critical point. So this gives a different mapping $G_{1}$. However, for a circle covering $h: S^{1} \rightarrow S^{1}$, any two extensions of $h$ in the unit disc as a branched covering such that $h^{-1}(0)=0$ are topologically conjugate by a homeomorphism $\psi: \bar{D} \rightarrow \bar{D}$ with $\left.\psi\right|_{S^{1} \cup\{0\}}=\mathrm{id}$. In our case, there is a homeomorphism $\Phi$ of the sphere, which is the identity on $S^{2}-H^{-n}(\mathcal{B})$, mapping the first choice of the marked orbit to the second one, such that $\Phi^{-1} \circ G_{1} \circ \Phi$ coincides with $G$ everywhere, except on $H^{-(n+1)}(\mathcal{B})-H^{-n}(\mathcal{B})$. The exceptional set is a disjoint union of finite discs away from $P_{G}$. So $\Phi^{-1} \circ G_{1} \circ \Phi$ and $G$ are isotopic rel $P_{G}$. Thus $G_{1}$ and $G$ are Thurston equivalent.

Now the mapping $s:[F] \rightarrow[G]$ is well defined.

II. Properties of s. Any rational map $R$ which is postcritically finite has no Levy cycle [Bielefeld et al. 1992], so $s([R])=[R]$.

Next, let $F, H, G, \mathcal{B}$ be as above. Since $P_{G} \cap$ $H^{-n}(\mathcal{B})$ is contained in the set of marked points, curves in $\partial H^{-n}(\mathcal{B})$ are peripheral for $G$. So for $\xi \in A_{G}$ and $\tilde{\Gamma}$ a representative of $\xi$ such that $\tilde{\Gamma} \cap$ $H^{-n}(\mathcal{B})=\varnothing$, we have $\left.G^{-1}\right|_{\tilde{\Gamma}}=\left.H^{-1}\right|_{\tilde{\Gamma}}$. So $\tilde{\Gamma}$ is again an obstruction for $H$ and then for $F$. It gives rise to a uniquely determined $\xi^{\prime} \in A_{F}$.

Now let $\xi^{\prime} \in A_{F}$, so $\xi^{\prime}$ is not smaller than any element of $\Sigma^{\prime}(F)$. There is a representative $\tilde{\Gamma}$ of $\xi^{\prime}$ such that $\tilde{\Gamma} \cap \mathcal{B}=\varnothing$. So $\tilde{\Gamma}$ is an obstruction for $H$. Now since $\tilde{\Gamma} \cdot \partial \mathcal{B}=0$, by Theorem 3.9 (with $\Lambda=\partial \mathcal{B}$ ), we have $\tilde{\Gamma} \cdot H^{-n}(\partial \mathcal{B})=0$. Moreover $\tilde{\Gamma}$ is not isotopically contained in $H^{-n}(\mathcal{B})$. So we can choose $\tilde{\Gamma}$ in its 
isotopy class such that $\tilde{\Gamma} \cap H^{-n}(\mathcal{B})=\varnothing$. In other words, $\tilde{\Gamma}$ is in the identical part of $H^{-1}$ and $G^{-1}$. As a consequence, $\lambda(\tilde{\Gamma}, G)=\lambda(\tilde{\Gamma}, H)=\lambda(\tilde{\Gamma}, F) \geq 1$ (this shows in particular that curves in $\tilde{\Gamma}$ are not peripheral for $G$ ). Thus $\tilde{\Gamma}$ is an irreducible obstruction for $G$, and gives rise to a uniquely determined $\xi \in A_{G}$.

Finally, let $\tilde{\Gamma}$ be a maximal removable Levy cycle of $G$ such that $\tilde{\Gamma} \cdot \Gamma=0$ for any irreducible obstruction $\Gamma$ of $G$ (i.e., $[\tilde{\Gamma}] \in \Sigma^{\prime}(G)$ ). It is again a removable Levy cycle for $F$ and gives rise to a $\xi^{\prime} \in A_{F}$, by the preceding paragraph. Moreover $\tilde{\Gamma} \cdot \Gamma=0$ for any irreducible obstruction $\Gamma$ of $F$. By definition of $\Sigma^{\prime}(F)$ and the fact that $\xi^{\prime} \notin \Sigma^{\prime}(F)$, there is a removable Levy cycle $\xi^{\prime \prime}$ for $F$ strictly larger than $\xi^{\prime}$. This contradicts the maximality of $\tilde{\Gamma}$ for $G$.

As a consequence, $s \circ s=s$.

Definition. Two branched coverings $F, F^{\prime}$ are weakly equivalent if $s([F])=s\left(\left[F^{\prime}\right]\right)$. We say that $F$ is weakly equivalent to a rational map $R$ if $s([F])=$ $[R]$.

Notation. We denote by $s(F)$ a representative of $s([F])$.

Corollary 3.13. If all irreducible obstructions for $F$ are removable Levy cycles smaller than or equal to an element of $\Sigma^{\prime}(F), F$ is weakly equivalent to a rational map. On the other hand, in case that $s(F)$ has a hyperbolic orbifold, if $F$ is weakly equivalent to a rational map then all irreducible obstructions for $F$ are removable Levy cycles smaller than or equal to an element of $\Sigma^{\prime}(F)$.

Proof. Set $G=s(F)$. In the first case $G$ has no irreducible obstructions; by Lemma 3.5 it has no Thurston obstructions. Therefore by Theorem $1.1 G$ is equivalent to a rational map. In the second case, $G$ has no Thurston obstructions (Theorem 1.1). We can then apply part (b) of the theorem above.

We conclude this section with applications to several problems about matings.

\section{F. A Criterion for a Branched Covering to Be a Mating}

Every mating is a branched covering. But many branched coverings can not be realized as a mating, for instance with mixed critical orbits. As an example, choose a degree two branched covering with one critical point periodic and the other falling eventually into the first orbit. But this mixed-orbit condition is far from sufficient, even in degree two case there are rational maps whose both critical points are periodic with disjoint orbits but which is not equivalent to a mating (see [Wittner 1986]). In fact the key point for a branched covering to be equivalent to a mating is that there is a curve is $S^{2}$ playing isotopically the role of the equator for a mating:

Theorem 3.14 [Thurston 1983; Levy 1985; Wittner 1986]. Let $F$ be a postcritically finite branched covering of degree $d$. Assume that $F$ has no degenerate Levy cycle. Then $F$ is equivalent to the mating of two polynomials $f, g$ if and only if there is a closed curve $\gamma \subset S^{2}-P_{F}$ such that $F^{-1}(\gamma)=\gamma^{\prime}$ is again a single closed curve and $\gamma^{\prime}$ is isotopic to $\gamma$ rel $P_{F}$ with the same orientation. Moreover given such a $\gamma$ the two polynomials $f, g$ are uniquely determined.

It happens also that there are several "equators" for the same branched covering. This phenomenon is called shared mating in [Wittner 1986]. Hence the mapping $f, g \mapsto f \Perp g$, from the space of pairs of monic polynomials of degree $d$ to the space of degree- $d$ branched coverings, is neither surjective nor injective.

\section{G. Levy Cycles around Periodic Ray Classes}

This part intends to complete the picture of Theorem 1.4 .

Lemma 3.15. Let $F$ be a mating. Let $[x]$ be a periodic ray class such that $[x]$ contains either a closed loop or at least two postcritical points. Then each boundary curve of a tubular neighborhood of $[x]$ generates a Levy cycle.

Proof. All we need to prove is that such a curve $\gamma$ is not peripheral (the periodicity and the degree-one property are guaranteed by that of $[x])$. But if $\gamma$ were peripheral, the disc component $D$ of $S^{2}-[x]$ containing $\gamma$ would be periodic, i.e. there would be an integer $k>0$ such that $F^{-k}(D)$ has a component which coincides with $D$, i.e. $F^{k}(D)=D$. But $D \cap E$ consists of finitely many intervals (where $E$ is the equator), and $F^{k}$ is expanding on $E$. So

$$
F^{k}(D \cap E) \neq D \cap E,
$$

which is a contradiction. 


\section{H. How to Find Removable Levy Cycles in a Mating}

Proposition 3.16. Let $F=f \Perp g$ be a postcritically finite mating. The set $\Sigma^{\prime}(F)$ consists of boundary curves of tubular neighborhoods of periodic cycles $\left\{\left[x_{1}\right],\left[x_{2}\right], \ldots,\left[x_{m}\right]\right\}$ of ray classes such that each $\left[x_{i}\right]$ contains at least two postcritical points and no ray class in $\bigcup_{n>0} F^{-n}\left(\left[x_{i}\right]\right)$ contains a closed loop. If $S^{2} / \sim_{\text {ray }}$ is homeomorphic to $S^{2}$ then $F$ has no non-removable Levy cycles. Moreover $f$ and $g$ are matable if all irreducible obstructions of $F$ are removable Levy cycles. And, in case that $s(F)$ has a hyperbolic orbifold, if $f$ and $g$ are matable then all irreducible obstructions of $F$ are removable Levy cycles.

Proof. We will only sketch the proof here. The details are left to the reader.

It is very easy to see that the boundary curves $\Gamma$ of a tubular neighborhood of such a periodic cycle of ray classes form a removable Levy cycle. To see that $\Gamma$ is maximal among removable Levy cycles, we apply Theorem 1.4. To see that $\Gamma$ is (geometrically) disjoint from any other Levy cycle we apply Lemma 3.15 and then Theorem 1.4. To see that every element of $\Sigma^{\prime}(F)$ is in this form we apply again Theorem 1.4.

Assume now that $F$ has a non-removable Levy cycle. Then the limit set of the cycle $\left\{\left[x_{1}\right], \ldots,\left[x_{m}\right]\right\}$ satisfies that for some $i$ and some $n$, the set $F^{-n}\left(\left[x_{i}\right]\right)$ contains a closed loop. So one of the ray classes contains a closed loop. Therefore $S_{F}^{2} / \sim_{\text {ray }}$ is not homeomorphic to $S^{2}$.

Recall that by definition $f$ and $g$ are matable if $F$ is weakly equivalent to a rational map, that is, if $s(F)$ is equivalent to a rational map.

Due to Theorem 1.4 and Lemma 3.15, if all irreducible obstructions of $F$ are removable Levy cycles, each of them must be smaller than or equal to an element of $\Sigma^{\prime}(F)$. So $F$ is weakly equivalent to a rational map, by Corollary 3.13.

On the other hand, in case that $s(F)$ has a hyperbolic orbifold, if $F$ is weakly equivalent to a rational map then by Corollary 3.13 again all irreducible obstructions of $F$ are removable Levy cycles.

Now we give an example of a maximal removable Levy cycle which is not geometrically disjoint from an irreducible obstruction. Consider $f$ to be a cubic polynomial with one fixed critical point and the second iterate $\beta$ of the other critical point fixed (so that the second critical point behaves like 0 for $\left.z^{2}-2\right)$. Consider now $F=f \Perp \tilde{f}$, with $\tilde{f}(z)=-f(-z)$. Then the ray class $[\beta]$ consists of two fixed rays $R(0)$ and $R(1 / 2)$. A curve around each ray is a maximal removable Levy cycle, but intersects always a curve around the other ray.

\section{PROOF OF THE RESULTS: FIRST PART}

If $F \in \mathcal{A}$ is postcritically finite, then it is easy to check (by definition of $s$ ) that $s(F) \in \mathcal{A}$ and $s(F)$ has a hyperbolic orbifold.

Proof of Theorem 2.6. Denote by $P$ one of $P_{3}, P_{4}$. Then $P$ is star-like, with $\alpha$ the unique branched point in its Hubbard tree (see Figure 1). Denote by $-\theta_{1},-\theta_{2},-\theta_{3}$ the external angles of $\alpha$. They form a single orbit by $\theta \mapsto 3 \theta$. According to Corollary 3.11, an irreducible obstruction for $g_{a} \Perp P$ is a Levy cycle $\Gamma$ with limit set $[\alpha]$, and, either $[\alpha]$ is a tree, or $R_{g_{a}}\left(\theta_{1}\right), R_{g_{a}}\left(\theta_{2}\right)$ and $R_{g_{a}}\left(\theta_{3}\right)$ all land at the same point in $J_{g_{a}}$ which is also a fixed point of $g_{a}$. This latter case does not occur for the following reasons: the map $g_{a}$ has a unique fixed point $\alpha^{\prime}$ having more than one external angles, and $\alpha^{\prime} \in[a, w]$. A simple calculation shows that the set of external rays of $\alpha^{\prime}$ is disjoint from either $\left[0, \frac{1}{3}\right]$ or $\left[\frac{2}{3}, 1\right]$. But $\left\{\theta_{1}, \theta_{2}, \theta_{3}\right\}$ intersects both $\left[0, \frac{1}{3}\right]$ and $\left[\frac{2}{3}, 1\right]$. So $\alpha^{\prime} \notin[\alpha]$.

As a consequence, $[\alpha]$ is a tree. Furthermore, by induction each component of $F^{-n}([\alpha])$ is again a tree and contains at most one critical value (that is, $a$, since the other two critical values $y$ and $z$ do not have external rays). Therefore each component of $F^{n+1}([\alpha])$ is a tree. Hence any irreducible obstruction is a removable Levy cycle surrounding $[\alpha]$. So $g_{a} \Perp P$ is weakly equivalent to a rational map (Proposition 3.16).

Proof of Theorem 2.5. The part D $\Longrightarrow \mathrm{A}$ is proved in Proposition 3.16 for general matings (recall that $s\left(g_{a} \Perp P_{2}\right)$ has always a hyperbolic orbifold).

$\mathrm{B} \Longrightarrow \mathrm{C}$. In this case the closed curve $R\left(\frac{5}{8}\right) \cup R\left(\frac{7}{8}\right)$ or a tubular neighborhood of it forms a good (thus non-removable) Levy cycle. $\mathrm{C} \Longrightarrow \mathrm{D}$ is trivial.

It remains to show $\mathrm{D} \Longrightarrow \mathrm{B}$. By Theorem 2.2 (to be proved later), the map $g_{a} \Perp P_{2}$ has a good Levy cycle with at most two curves. Its limit set $X_{0}$ is 
contained in a ray class and is a simple closed curve with two fixed points (Theorem 1.4). Therefore $\alpha \in$ $X_{0}$, where $\alpha$ is the fixed point in $H_{P_{2}}$ (see Figure 1). The two external angles of $\alpha$ are $-\frac{5}{8},-\frac{7}{8}$. No other two-periodic rays land at a point of $H_{P_{2}}$. Similar to the proof of Corollary 3.11, one can show that either $R_{a}\left(\frac{5}{8}\right)$ and $R_{a}\left(\frac{7}{8}\right)$ land at the same point or $[\alpha]$ is a tree. But the latter case does not occur since $X_{0} \subset[\alpha]$. So $\frac{5}{8} \sim_{g_{a}} \frac{7}{8}$, proving B.

The rest of this section is devoted to the proof of the equivalence $1 \Leftrightarrow 2$ in Theorem 2.4. First we need to study in more detail the ray-equivalence relation for $g_{a} \Perp P_{1}$.

According to Douady and Hubbard [1984], for any postcritically finite polynomial $f$ with $K$ as its filledin Julia set, there is a way to define a (unique) regular arc $[u, v] \subset K$ for any pair of points in $K$ so that the convex hull $\left[u_{1}, \ldots, u_{l}\right] \subset K$ of any finite subset is a topologically finite tree, and $f\left(\left[u_{1}, \ldots, u_{l}\right]\right)=$ $\left[f\left(u_{1}\right), \ldots, f\left(u_{l}\right), f\left(w_{1}\right), \ldots, f\left(w_{k}\right)\right]$, where $w_{1}, \ldots$, $w_{k}$ are the critical points of $f$ in $\left[u_{1}, \ldots, u_{l}\right]$. The Hubbard tree is precisely the convex hull of the postcritical set.

As in Section $2 \mathrm{C}$, set $P=P_{1}$, and for $i=a, P$, denote by $\gamma_{i}(\theta)$ the landing point of $R_{i}(\theta)$ on $K_{i}$. Let $F=g_{a} \Perp P$.

Set $\hat{H}_{a}=\left[H_{a}, \beta_{0}, \beta_{1}, \beta_{2}\right]$ with $\beta_{i}=\gamma_{a}(i / 3)$ and $\hat{H}_{P}=\left[H_{P}, \beta_{0}^{\prime}, \beta_{1}^{\prime}, \beta_{2}^{\prime}\right]$ with $\beta_{i}^{\prime}=\gamma_{P}(i / 3)$. These are the extended Hubbard trees. Note that $\hat{H}_{P}=H_{P} \cup$ $\left[y, \beta_{1}^{\prime}\right] \cup\left[y, \beta_{2}^{\prime}\right] \cup\left[\beta_{0}^{\prime}, z\right]$ (see Figure 3). For $i=a, P$, we have $F\left(H_{i}\right) \subset H_{i}$ and $F\left(\hat{H}_{i}\right) \subset \hat{H}_{i}$.

Note that each nontrivial ray class $[u]$ is completely determined by $[u] \cap\left(J_{a} \sqcup J_{P}\right)$. For example, the extremities of $[u]$ are points in $[u] \cap\left(J_{a} \sqcup J_{P}\right)$ having only one external angle. The other points in $[u] \cap\left(J_{a} \sqcup J_{P}\right)$ have at least two external rays. For this reason, we give

Definition (simple and multiple point). A point $u$ in $J_{a} \sqcup J_{P} \subset S_{g_{a}, P}^{2}$ is a simple point if $u$ has only one external angle, and a multiple point if $u$ has at least two external angles.

Lemma 4.1. If $u$ is multiple and $u \neq w, F(u)$ is also multiple. If $w \notin[u]$, then $F:[u] \rightarrow[F(u)]$ is a homeomorphism.

Proof. In $u=x$ or $y$ it has no external rays and $[u]$ is trivial. If $u$ is not a critical point of $F$, the mapping
$F$ is a local homeomorphism, and $F$ sends external rays to external rays. So $F(u)$ has the same number of external angles as $u$. The rest follows.

Lemma 4.2. For $i=a, P$, there is an integer $m_{i}$ such that for any $u \in J_{i}$, the number of external angles $\#>u$ is bounded by $m_{i}$. Moreover $m_{P}=2$ and no point in $H_{P}$ has external angles in $\left[\frac{1}{3}, \frac{2}{3}\right]$.

Proof. For a multiple point $u \in J_{P}$, since all critical points of $P$ are in $\operatorname{int}\left(K_{P}\right)$, the orbit of $u$ contains no critical points. There is $k$ such that $F^{k}(u) \in H_{P}$ (Proposition 3.1) and $F^{k}$ is a homeomorphism in a neighborhood of $u$. So $\#>u=\#>F^{k}(u)$. Since

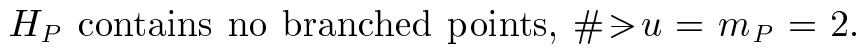
Moreover since $\left[\beta_{1}^{\prime}, \beta_{2}^{\prime}\right] \cap H_{P}=\{y\}$ and $y$ has no external rays (Figure 3 ), no point in $H_{P}$ has angle in $\left[\frac{1}{3}, \frac{2}{3}\right]$.

It is known that there is $m_{a}$ such that $\# \rightarrow u \leq m_{a}$ for any $u \in H_{a}$. For a multiple point $u \in J_{a}$, either there is a unique $k$ such that $F^{k}(u)=w$ or the orbit of $u$ does not contain $w$ and there is $k$ such that $F^{k}(u) \in H_{a}$ (Proposition 3.1). In both cases $\#>u=\#>F^{k}(u) \leq m_{a}$.

Lemma 4.3. Every multiple point $u$ in $J_{a}$ has a forward image in $[w, a]$.

Proof. Assume that none of the iterates of $u$ meets $w$ (otherwise the lemma is trivial). Iterate $u$ enough times so that the set of external angles of $F^{n}(u)$ meets at least two components of $\mathbf{T}-\left\{0, \frac{1}{3}, \frac{2}{3}\right\}$. Then $F^{n}(u) \in\left[\beta_{0}, w\right] \cup\left[\beta_{1}, w\right] \cup\left[\beta_{2}, w\right]$. We have $F\left(\left[\beta_{i}, w\right]\right)=\left[\beta_{0}, w\right] \cup[w, a]$, for $i=0,1,2$, and $F$ : $\left[\beta_{0}, w\right] \rightarrow\left[\beta_{0}, a\right]$ is expanding. So there is $n^{\prime} \geq n$ such that $F^{n^{\prime}}(u) \in[w, a]$.

Proposition 4.4. Each ray class $[u]$ for $F$ contains at most $m_{a}+1$ points of $J_{a}$ and no closed loops.

Proof. If all points in $[u] \cap J_{a}$ are simple we are done since a point in $[u] \cap J_{P}$ has at most two external rays.

Claim. Suppose that $u$ is a multiple point of $J_{a}$ and that $F^{n}(u) \neq w$ for all $n$. Let $\theta, \theta^{\prime}$ be two distinct angles of $u$ and $v=\gamma_{P}(-\theta), v^{\prime}=\gamma_{P}\left(-\theta^{\prime}\right)$. Then at least one of $v, v^{\prime}$ is a simple point. A consequence of this is that, if there is $\eta \in \angle u$ with $\gamma_{P}(-\eta)$ multiple, then $\gamma_{P}\left(-\eta^{\prime}\right)$ is simple for any $\eta^{\prime} \in \angle u$ with $\eta^{\prime} \neq \eta$. 


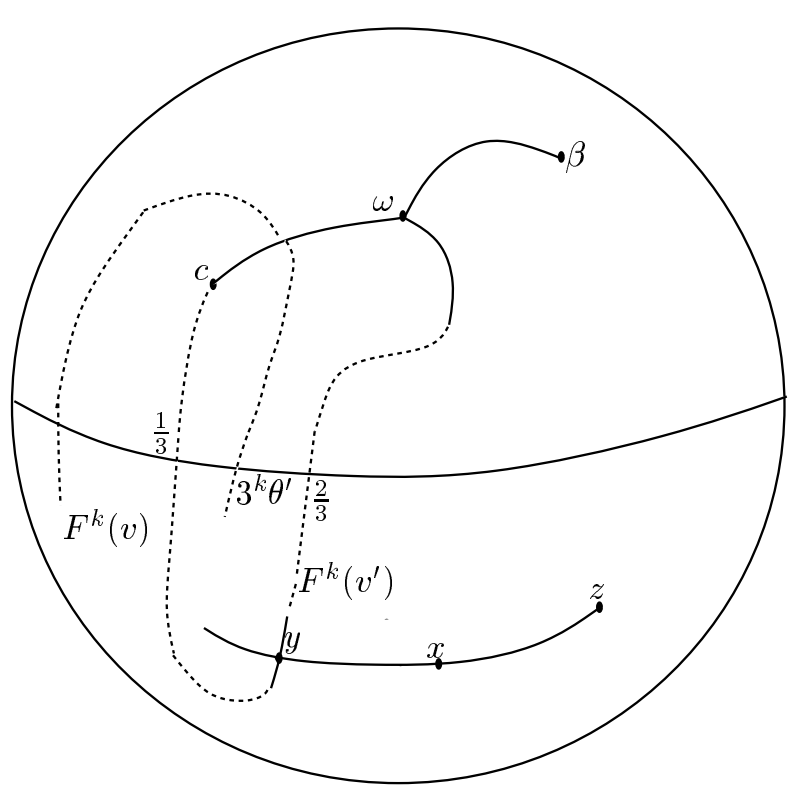

FIGURE 5. No point in $H_{P}$ has an external angle in $\left[\frac{1}{3}, \frac{2}{3}\right]$.

Suppose by contradiction that both $v$ and $v^{\prime}$ are multiple.

Note that $w$ disconnects $\hat{H}_{a}$ into three components $W_{0}, W_{1}, W_{2}$ with $\beta_{i} \in W_{i}$, and $[w, a]-\{w\}$ is in one of $W_{1}, W_{2}$.

Since $F^{n}(u) \neq w$ for all $n, F^{n}(u), F^{n}(v)$ and $F^{n}\left(v^{\prime}\right)$ are multiple and $F^{n}(v) \neq F^{n}\left(v^{\prime}\right)$ for all $n$. Take $k$ large enough such that $F^{k}(u) \in[w, a]-\{w\}$ (see Lemma 4.3) and such that $F^{n}(u) \in H_{a}$ and $F^{n}(v)$, $F^{n}\left(v^{\prime}\right) \in H_{P}$ for all $n \geq k$ (Proposition 3.1). We may assume $k=0$. Now there is $l \geq 0$ minimal such that $\left\{3^{l} \theta, 3^{l} \theta^{\prime}\right\}$ intersects at least two components of $\mathbf{T}-\left\{0, \frac{1}{3}, \frac{2}{3}\right\}$. In case $l=0$, we have $u \in[w, a]-\{w\}$ so $u \in[w, a] \cap\left[\beta_{i}, w\right]$ for $i=1$ or 2. Therefore one of the angle, say $\theta$, is in $\left[\frac{1}{3}, \frac{2}{3}\right]$. So $-\theta \in\left[\frac{1}{3}, \frac{2}{3}\right]$. But $v=\gamma_{P}(-\theta)$ is in $H_{P}$. This is impossible since no point in $H_{P}$ has angles in $\left[\frac{1}{3}, \frac{2}{3}\right]$ (Lemma 4.2; see also Figure 5). In case $l>0$, if $F^{l}(u) \in W_{1} \cup W_{2}$, then one of the angles, say $3^{l} \theta$ is in $\left[\frac{1}{3}, \frac{2}{3}\right]$ with $F^{l}(v) \in H_{P}$. Again this is impossible. Assume now $l>0$ and $F^{l}(u) \in W_{0}$. We will show this does not occur. In $S_{q_{a}, P}^{2}$, the two rays $R_{a}\left(3^{l} \theta\right)$ and $R_{a}\left(3^{l} \theta^{\prime}\right)$ together with a portion of the equator form a Jordan curve. Denote by $D$ the disc bounded by this curve containing $\beta_{0}$. Then $F^{-1} D$ has three discs each contains one of $\beta_{0}, \beta_{1}, \beta_{2}$. So the curve $R_{a}\left(3^{l-1} \theta\right) \cup F^{l-1}(u) \cup R_{a}\left(3^{l-1} \theta^{\prime}\right)$ together with a portion of the equator separates already $\left\{\beta_{0}, \beta_{1}, \beta_{2}\right\}$.
This contradicts the fact that $l$ is minimal. This ends the proof of the claim.

Now consider the critical value class $[a]$. If it is nontrivial $a$ is eventually periodic (Misiurewicz case) and is never mapped to $w$.

For any two distinct angles $\theta_{1}, \theta_{2}$ of $a$ we have $\gamma_{P}\left(-\theta_{1}\right) \neq \gamma_{P}\left(-\theta_{2}\right)$ otherwise $\gamma_{P}\left(-\theta_{1}\right)$ and $\gamma_{P}\left(-\theta_{1}\right)$ are both multiple contradicting the above claim.

Let $\theta$ be an angle of $a$. The point $\gamma_{P}(-\theta)$ is either simple or it has only one other angle $-\eta$. In the latter case let $u^{\prime}=\gamma_{a}(\eta)$. Since $\eta$ has the same preperiod as $\theta, u^{\prime}$ is not mapped to $w$ under the iterates of $F$. If $u^{\prime}$ is multiple, for any $\theta^{\prime} \neq \eta$ external angle of $u^{\prime}$, by the above claim the point $\gamma_{P}\left(\theta^{\prime}\right)$ is simple. In any case $\#\left([a] \cap J_{a}\right) \leq 1+\#>a$, and $[a]$ contains no loop.

An easy consequence is that \# $\left([w] \cap J_{a}\right) \leq 1+$ $\#>w=1+3 \#>a, \#\left([u] \cap J_{a}\right)=\#\left([w] \cap J_{a}\right)$ if $F^{l}(u)=w$ for some $l \geq 0$, and these ray classes don't contain closed loops.

Now assume $[u]$ contains no preimage of $w$. We claim that $\#\left([u] \cap J_{a}\right) \leq 2$ and $[u]$ contains no closed loop. Again this is easy if all points in $[u] \cap J_{a}$ are simple. Assume that $u$ is a multiple point in $J_{a}$. Then by the above claim there is at most one external angle of $u$, say $\theta$, such that $\gamma_{P}(-\theta)$ is multiple, and this point has at most one more angle $-\eta$. Now the point $u^{\prime}=\gamma_{a}(\eta)$ satisfies the condition of the claim. So any other angle than $\eta$ of $u^{\prime}$ must lead to a simple point in $J_{P}$. As a consequence $[u] \cap J_{a}=\left\{u, u^{\prime}\right\}$ and $[u]$ contains no loops.

Corollary 4.5. Each ray class of $F$ intersects the equator $E$ at at most $m_{a}\left(m_{a}+1\right)$ points.

Proof. Each point of $[u] \cap J_{a}$ has at most $m_{a}$ external rays and $[u] \cap J_{a}$ has at most $m_{a}+1$ points.

The next result is part of Theorem 2.4.

Corollary 4.6. The quotient $S_{g_{a}, P}^{2} / \sim_{\text {ray }}$ is again a sphere.

Proof. The previous two results put us in the situation of Proposition 3.2 and Corollary 3.3.

Proof of Theorem 2.4, part $1 \Leftrightarrow 2$. We need to show that $g_{a}$ and $P_{1}$ are not matable if and only if $g_{a} \Perp P_{1}$ has a nested obstruction which is not a Levy cycle. The "if" part follows from Proposition 3.16. For the other direction, since $S_{g_{a}, P}^{2} / \sim_{\text {ray }}$ is a sphere, the 
Levy cycles of $g_{a} \Perp P_{1}$ are all removable (Proposition 3.16). Now we can apply Theorem 2.2 (to be proved later) to conclude that $g_{a} \Perp P_{1}$ must have a nested obstruction.

\section{PROOF OF THE RESULTS: SECOND PART}

Proof of Theorem 2.2. It is not difficult from the following argument to see that the three cases are mutually distinct.

Recall that $\lambda(\Gamma) \geq 1$ by assumption. We will apply repeatedly Lemma 3.6.

Step 1. Suppose there is a disc-component $D$ of $S^{2}-$ $\Gamma$ such that $D \cap\{x, y, z\}=\varnothing$. Then each connected component of $F^{-n}(D)$ is disjoint from $\{x, y, z\}$, so contains at most one critical value. So by induction on $n$ all preimage components of $D$ are discs. By Proposition 3.7, $\Gamma$ is a removable Levy cycle.

Step 2. Suppose that there are exactly three disccomponents $D_{x}, D_{y}, D_{z}$ of $S^{2}-\Gamma$, with $x \in D_{x}$, $y \in D_{y}, z \in D_{z}$. We claim that this is impossible.

If $a \notin D_{y} \cup D_{z}$, each component of $S^{2}-\Gamma$ contains at most one critical value. By Proposition 3.7, we have $\Gamma=\left\{\gamma_{x}, \gamma_{y}, \gamma_{z}\right\}$. But in this case $\lambda(\Gamma)<1$ (by looking at the degree).

Now assume $a \in D_{z}$. Then $F^{-1}\left(D_{z}\right)$ is connected, containing $y$, but none of $x, z$. So $F^{-1}\left(D_{z}\right)$ is isotopically contained in $D_{y}$. As a consequence, one connected component of $F^{-1}\left(\gamma_{z}\right)$ is isotopic to $\gamma_{y}$, the other is not in $\Gamma$. Thus $\Gamma=\left\{\gamma_{x}, \gamma_{y}, \gamma_{z}\right\}$, and, by looking at the degree, $\lambda(\Gamma)<1$. The case $a \in D_{y}$ is similar.

Step 3. Suppose that there are exactly two disccomponents $D_{1}, D_{2}$ with $\#\left(D_{1} \cap\{x, y, z\}\right)=1$ and $\#\left(D_{2} \cap\{x, y, z\}\right)=2$. We claim then $\Gamma$ is a good Levy cycle with at most two curves.

Proof. Set $\gamma_{i}=\partial D_{i}$. We claim at first that $a \in D_{1}$ and $D_{1} \cap\{x, y, z\} \subset\{y, z\}$. If not, $x \in D_{1}$ and $D_{1}$ contains at most one critical value. So $F^{-1}\left(D_{1}\right)$ are discs. One of them contains $z$, but not $x, y$, the others are disjoint from $\{x, y, z\}$. Thus no curve in $F^{-1}\left(\partial D_{1}\right)$ is isotopic to a curve in $\Gamma$. This contradicts the irreducibility.

One possibility is $a, z \in D_{1}$ and $x, y \in D_{2}$. In this case, $F^{-1}\left(D_{2}\right)$ consists of two discs one of which is strictly contained in $D_{2}$, so the other must be isotopic to $D_{1}$, with degree 1 . On the other hand, $F^{-1}\left(D_{1}\right)$ is an annulus isotopically contained in $D_{2}$.
The connected component $\gamma^{\prime}$ of $F^{-1}\left(\gamma_{1}\right)$ separating $z$ and $y$ is isotopic to $\gamma_{2}$, and $\operatorname{deg}\left(F: \gamma^{\prime} \rightarrow \gamma_{1}\right)=$ 1. Thus $\Gamma=\left\{\gamma_{1}, \gamma_{2}\right\}$ is a Levy cycle. Let $A$ be the complement of $\bar{D}_{1} \cup \bar{D}_{2}$. Either $A=\varnothing$ or a component of $F^{-1}(A)$ is isotopic to $A$ of degree 1 . In any case $\Gamma$ is a good Levy cycle with at most two curves.

The other possibility is $a, y \in D_{1}$ and $x, z \in D_{2}$. A similar analysis will show that $\Gamma=\left\{\gamma_{1}, \gamma_{2}\right\}$ with $\lambda(\Gamma)=1 / 2$, which is excluded by the hypothesis.

Step 4. Suppose that there are exactly two disccomponents $D_{1}, D_{2}$ with

$$
\#\left(D_{1} \cap\{x, y, z\}\right)=\#\left(D_{2} \cap\{x, y, z\}\right)=1 .
$$

We claim that either $a, y \in D_{1}$ and $z \in D_{2}$ or $a, z \in$ $D_{1}$ and $x \in D_{2}$. In the latter case, some preimages of $\Gamma$ contain a good Levy cycle with at most two curves.

Proof. Similarly to step 2 above, one of the two discs, say $D_{1}$, must contain two critical values. One is $a$, the other is one of $y, z$. So $F^{-1}\left(D_{2}\right)$ are discs. So at least one component $D^{\prime}$ of $F^{-1}\left(D_{2}\right)$ is isotopic to one of $D_{1}, D_{2}$. It can not be isotopic to $D_{2}$ because of the periodic cycle $\{x, y, z\}$, so it must be isotopic to $D_{1}$. Thus if $y \in D_{1}$, then $z \in D_{2}$; if $z \in D_{1}$ then $x \in D_{2}$.

Suppose $x \in D_{2}$. Set $\gamma_{1}=\partial D_{1}$ and $\gamma_{2}=\partial D_{2}$. Complete $\Gamma$ into an $F$-invariant multicurve $\Gamma^{\prime}$, as in the proof of Lemma 3.5. Define a subset

$$
S_{1}=\left\{\gamma_{1}\right\} \cup\left\{\gamma \in \Gamma^{\prime} \mid \gamma \text { separates } \gamma_{1} \text { and }\{y, x\}\right\}
$$

(see the remark below). This set has a natural order. For any $\gamma \in S_{1}$, we have $F^{-1}(\gamma)=\gamma^{*} \cup \gamma^{* *}$, with $\left[\gamma^{*}\right]_{\Gamma^{\prime}} \in S_{1},\left[\gamma^{* *}\right]_{\Gamma^{\prime}} \notin S_{1}, \operatorname{deg}\left(F: \gamma^{*} \rightarrow \gamma\right)=1$, and the mapping $\gamma \mapsto\left[\gamma^{*}\right]_{\Gamma^{\prime}}, S_{1} \rightarrow S_{1}$ is weakly decreasing. So there is a periodic cycle of period at most 2 .

Step 5. Now assume $a, y \in D_{1}, z \in D_{2}$ and $x \notin$ $D_{1} \cup D_{2}$. By definition, $\Gamma$ is a nested obstruction. We just need to show that it's not a Levy cycle. This is part of Proposition 2.3.

Remark. We say that a curve $\gamma \in S^{2}$ separates two sets $U$ and $V$ if $U$ is contained in one component of $S^{2}-\gamma$ and $V$ is contained in the other component of $S^{2}-\gamma$. Recall that $[\eta]_{\Gamma}$ denotes the curve in $\Gamma$ isotopic to $\eta$, and $[\eta]_{\Gamma}=0$ by convention, if no such curve exists. 
Proof of Proposition 2.3. Assume at first $\lambda\left(\Gamma^{\prime}\right) \geq 1$. Let $\Gamma \subset \Gamma^{\prime}$ be an irreducible obstruction. Denote by $D_{ \pm}, A_{0}$ the sets $D_{ \pm}(\Gamma), A_{0}(\Gamma)$ respectively. An application of Lemma 3.6 would show that, up to isotopy, $D_{+}$is a component of $F^{-1}\left(D_{-}\right), A_{0}$ does not contain curves of $\Gamma$ and separates $D_{+}$and $D_{-}$.

Let $B_{ \pm}$be the discs of $S^{2}-\bar{A}_{0}$ containing $D_{ \pm}$(up to isotopy) respectively. Note that $x \in A_{0}, z \in B_{-}$, $y \in B_{+}$and $B_{+} \cup B_{-}=F^{-1}\left(S^{2}-\bar{D}_{+}\right)$.

We decompose $\Gamma$ into $S_{+} \sqcup S_{-}$with

$$
\begin{aligned}
& S_{+}=\{\gamma \in \Gamma \mid \gamma \text { separates }\{a, y\} \text { and }\{x, z\}\}, \\
& S_{-}=\{\gamma \in \Gamma \mid \gamma \text { separates }\{a, y, x\} \text { and }\{z\}\} .
\end{aligned}
$$

Take $\gamma \in \Gamma$. Denote by $\Delta$ the component of $S^{2}-$ $\gamma$ containing $z$, and by $\Delta_{ \pm}$the two components of $F^{-1}(\Delta)$, with $\operatorname{deg}\left(F: \Delta_{-} \rightarrow \Delta\right)=1$ and $\operatorname{deg}(F:$ $\left.\Delta_{+} \rightarrow \Delta\right)=2$. Then $\Delta_{ \pm}$are discs. Moreover, for $\gamma_{ \pm}=\partial \Delta_{ \pm}$we have $\operatorname{deg}\left(F: \gamma_{-} \rightarrow \gamma\right)=1$ and $\operatorname{deg}\left(F: \gamma_{+} \rightarrow \gamma\right)=2$.

For $\gamma \in \Gamma$, if $\gamma_{-}$is in $\Gamma$ then it is in $S_{-}$, and if $\gamma_{+}$ is in $\Gamma$ then it is in $S_{+}$. Moreover if $\gamma \in S_{-}$, then $\gamma_{-}$is not in $\Gamma$, since $z \notin \Delta_{-}$. Clearly $\Gamma$ does not contain a Levy cycle. Let

$$
v=\sum_{\gamma \in \Gamma} c_{\gamma} \gamma
$$

be a positive eigenvector (i.e. every $c_{\gamma}$ is positive) of $F_{\Gamma}$ with the eigenvalue $\lambda=\lambda(\Gamma)$. Such a positive eigenvector exists, since by the assumption $\Gamma$ is an irreducible multicurve. Write

$$
v_{i}=\sum_{\gamma \in S_{i}} c_{\gamma} \gamma, \quad\left|v_{i}\right|=\sum_{\gamma \in S_{i}} c_{\gamma}
$$

where $i=+,-$. Using the notation above, we have

$$
\begin{aligned}
& F_{\Gamma}\left(v_{+}\right)=\sum_{\gamma \in S_{+}} c_{\gamma}\left[\gamma_{-}\right]_{\Gamma}+\sum_{\gamma \in S_{+}} \frac{1}{2} c_{\gamma}\left[\gamma_{+}\right]_{\Gamma} \\
& F_{\Gamma}\left(v_{-}\right)=\sum_{\gamma \in S_{-}} \frac{1}{2} c_{\gamma}\left[\gamma_{+}\right]_{\Gamma}
\end{aligned}
$$

and

$$
\begin{array}{r}
\sum_{\gamma \in S_{+}} \frac{1}{2} c_{\gamma}\left[\gamma_{+}\right]_{\Gamma}+\sum_{\gamma \in S_{-}} \frac{1}{2} c_{\gamma}\left[\gamma_{+}\right]_{\Gamma}=\lambda v_{+} \\
\sum_{\gamma \in S_{+}} c_{\gamma}\left[\gamma_{-}\right]_{\Gamma}=\lambda v_{-}
\end{array}
$$

Hence

$$
\left.\begin{array}{rl}
\frac{1}{2}\left(\left|v_{+}\right|+\left|v_{-}\right|\right) & =\sum_{\gamma \in S_{+}} \frac{1}{2} c_{\gamma}+\sum_{\gamma \in S_{-}} \frac{1}{2} c_{\gamma} \\
& \geq \lambda \sum_{\gamma \in S_{+}} c_{\gamma}=\lambda\left|v_{+}\right|, \\
\left|v_{+}\right| & =\sum_{\gamma \in S_{+}} c_{\gamma} \\
& \geq \lambda \sum_{\gamma \in S_{-}} c_{\gamma}=\lambda\left|v_{-}\right| .
\end{array}\right\}
$$

A necessary and sufficient condition for both inequalities to be equalities is that

(*) for $\gamma \in S_{+}$, both $\gamma_{+}$and $\gamma_{-}$are isotopic to curves in $\Gamma$, and for $\gamma \in S_{-}, \gamma_{+}$is isotopic to a curve in $\Gamma$.

Adding two times the first inequality in $(5-1)$ to the second, we obtain

$$
2\left|v_{+}\right|+\left|v_{-}\right| \geq \lambda\left(2\left|v_{+}\right|+\left|v_{-}\right|\right) .
$$

Hence $\lambda \leq 1$. But $\lambda \geq 1$ by assumption. It follows that $\lambda=1$, and equalities hold in $(5-1)$, proving $(*)$. Applying $(*)$ to $\partial B_{+}$, which is a component of $\partial A_{0}$, we conclude that $\left(\partial B_{+}\right)^{-}$is isotopic to a curve in $\Gamma$, therefore separates $A_{0}$ from $D_{-}$.

We turn to the second half of the proposition. Let $\Gamma$ be a submulticurve of $\Gamma^{\prime}$ with the given properties. Define

$$
\begin{aligned}
& S_{+}=\left\{\gamma \in \Gamma \mid \gamma \text { separates } A_{0}(\Gamma) \text { and } D_{+}(\Gamma)\right\}, \\
& S_{-}=\left\{\gamma \in \Gamma \mid \gamma \text { separates } A_{0}(\Gamma) \text { and } D_{-}(\Gamma)\right\} .
\end{aligned}
$$

Then $\Gamma=S_{+} \sqcup S_{-}$.

By assumption, For $\gamma \in S_{+}$, we have $F^{-1}(\gamma)=$ $\gamma_{+} \cup \gamma_{-}$, with $\gamma_{+} \in S_{+}, \operatorname{deg}\left(F: \gamma_{+} \rightarrow \gamma\right)=2$ and $\gamma_{-} \in S_{-}, \operatorname{deg}\left(F: \gamma_{-} \rightarrow \gamma\right)=1$.

For $\gamma \in S_{-}$, we have $F^{-1}(\gamma)=\gamma_{+} \cup \gamma_{-}$, with $\gamma_{+} \in S_{+}, \operatorname{deg}\left(F: \gamma_{+} \rightarrow \gamma\right)=2$ and $\gamma_{-} \notin \Gamma$.

Define $h: \mathbb{R}^{\Gamma} \rightarrow \mathbb{R}^{2}$ by $h(\gamma)=\left(\begin{array}{l}1 \\ 0\end{array}\right)$ if $\gamma \in S_{+}$and $h(\gamma)=\left(\begin{array}{l}0 \\ 1\end{array}\right)$ if $\gamma \in S_{-}$. Define $G, G_{\#}: \mathbb{R}^{2} \rightarrow \mathbb{R}^{2}$ to be the linear maps with matrices

$$
\left(\begin{array}{cc}
\frac{1}{2} & \frac{1}{2} \\
1 & 0
\end{array}\right) \text { and }\left(\begin{array}{ll}
1 & 1 \\
1 & 0
\end{array}\right),
$$

respectively. Then one can check easily that $h \circ F_{\Gamma}=$ $G \circ h$ and $h \circ F_{\#, \Gamma}=G_{\#} \circ h$. Set $\lambda=\lambda_{\Gamma}$. Let $v$ be a nonnegative nonzero eigenvector of $F_{\Gamma}$ for $\lambda$, that is: $F_{\Gamma}(v)=\lambda \cdot v$. Hence $\lambda \cdot h(v)=G(h(v))$. Since $h$ has only nonnegative coefficients, $h(v)$ is also nonnegative nonzero. So $\lambda$ is also a positive eigenvalue for $G$. By looking at left eigenvectors and the fact 
that $h$ is surjective every eigenvalue of $G$ is also an eigenvalue of $F_{\Gamma}$. As a consequence they have the same leading eigenvalue, which is 1 . Similarly, $G_{\#}$ and $F_{\#, \Gamma}$ have the same leading eigenvalue, which is $\frac{1}{2}(1+\sqrt{5})$.

Proof of Theorem 2.4, $3 \Rightarrow 2$. We start by introducing some notation. Assume that $g_{a}$ is postcritically finite. We define $\theta^{ \pm}=\theta^{ \pm}(a)$ with $\theta^{-}<\theta^{+}$to be two angles such that the corresponding two external rays land at the same point $b=b(a)$ and their union separates $a$ from the rest of the critical orbit. If $w$ is periodic, we take $b$ to be the unique periodic point on the boundary of the immediate attracting basin of $a$ which has at least two arguments and whose period divides that of $a$. If $w$ is strictly preperiodic, we take $b$ a point arbitrarily close to $a$ having two arguments $\theta^{-}<\theta^{+}$with the required property. It is known that such a point $b$ always exists, and once $b$ is chosen the two arguments $\theta^{-}<\theta^{+}$are uniquely determined.

For $i=0,1,2$, denote by $\beta_{i}$ the point in $J_{a}$ with external angle $i / 3$. Let

$$
\tilde{H}=g_{a}^{-1}\left[H_{a}, \beta_{0}, \beta_{1}, \beta_{2}\right],
$$

where $H_{a}$ is the Hubbard tree and $\left[H_{a}, \beta_{0}, \beta_{1}, \beta_{2}\right]$ denotes the convex hull of $H_{a}$ and $\beta_{i}$ (the extended Hubbard tree).

Note that $w$ disconnects $\tilde{H}$ into three components $W_{0}, W_{1}, W_{2}$, numbered in a way such that $\beta_{i} \in W_{i}$. We have $a \in W_{1} \cup W_{2}$. Since $g_{a} \Perp P_{1}$ and $g_{\bar{a}} \Perp P_{1}$ are conjugate by an orientation reversing homeomorphism, one has a Thurston obstruction if and only if the other one has. So replace $g_{a}$ by $g_{\bar{a}}$ if necessary, we may assume in the following $a \in W_{1}$.

For any $z \in\left[H_{a}, \beta_{0}, \beta_{1}, \beta_{2}\right]-\{a\}$, denote by $z_{i}$ the point $g_{a}^{-1}(z) \cap W_{i}$. For $b$ the landing point of rays of angle $\theta^{ \pm}$, we have $b_{i} \in\left[H_{a}, \beta_{0}, \beta_{1}, \beta_{2}\right] \cap W_{i}$, for $i=0,1,2$. Furthermore $b_{i 0} \in \tilde{H}$. Also $w_{0} \in W_{0}$, and $w_{0}$ is the unique point in $\left[w, \beta_{0}\right]$ which is mapped to $w$. We may choose $b$ (and therefore $\theta^{ \pm}$) so that the $b_{i}$ 's are not separated from $w$ in $J_{a}$ (or $H_{a}$ ) by any points in the forward orbit of $a$, and similarly for the $b_{i 0}$ 's and $w_{0}$. The situation is this:

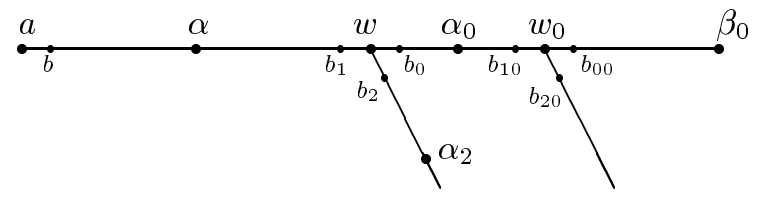

First assume that $\beta_{0}, g_{a}(a) \in W_{0}$ and $g_{a}^{3}$ is renormalizable. In particular, $\alpha$, the fixed point in $[a, w]$, is not in the postcritical set, and has external angles $\frac{1}{8}$ and $\frac{3}{8}$. Moreover the point $\alpha_{2}$ does not separate the period-three orbit of the renormalized Julia set (the small Julia sets). So we can define the curves $\delta_{1}, \delta_{2}$ exactly in the same way as in Section $2 \mathrm{C}$. Since $\delta_{1}$ does not meet the small Julia sets, no preimages of it does. Therefore $\delta_{3}, \delta_{5}$ as in Section $2 \mathrm{C}$ are homotopic to $\delta_{1}$. So $\delta_{1}, \delta_{2}$ form a nested obstruction.

Now consider the case where $\beta_{0}, g_{a}(a), g_{a}^{2}(a) \in$ $W_{0}$. One can choose $\theta^{ \pm}$so that neither $b_{10}$ nor $b_{20}$ belong to $\left[g_{a}(b), g_{a}(a)\right]$.

Recall from Section $2 \mathrm{C}$ that for $P_{1}$ the points $x_{*}, y_{*}, z_{*}$ denote the roots of basins of attraction of $x, y, z$ respectively, and $>y_{*}=\left\{\frac{4}{13}, \frac{9}{13}\right\},>z_{*}=$ $\left\{\frac{1}{13}, \frac{12}{13}\right\}$ (see Figure 3).

In $S_{g_{a}, P_{1}}^{2}$, we are going to modify the subsets

$$
\begin{aligned}
& \gamma_{-}^{\prime}:=R_{a}\left(3 \theta^{+}\right) \cup R_{a}\left(3 \theta^{-}\right) \cup\left\{g_{a}(b)\right\} \cup R_{P_{1}}\left(\frac{1}{13}\right) \\
& \cup R_{P_{1}}\left(\frac{12}{13}\right) \cup\left\{z_{*}\right\}, \\
& \gamma_{+}^{\prime}:=R_{a}\left(\theta^{+}\right) \cup R_{a}\left(\theta^{-}\right) \cup\{b\} \cup R_{P_{1}}\left(\frac{4}{13}\right) \cup R_{P_{1}}\left(\frac{9}{13}\right) \cup\left\{y_{*}\right\}
\end{aligned}
$$

in a neighborhood of the equator, so that they become two Jordan curves $\gamma_{-}$and $\gamma_{+}$whose homotopy classes rel $P_{F}$ do not depend on the modification.

To do this first we take a closed annular neighborhood $N$ of the equator of $S_{g_{a}, P_{1}}^{2}$ bounded by an equipotential $e^{u}$ of $g_{a}$ and an equipotential $e^{l}$ of $P_{1}$ (here the subscripts $u$ and $l$ mean "upper" and "lower" boundary of $N)$. We denote by $(\theta, u)$ the point in $e^{u}$ with angle $\theta$. Define $(\theta, l)$ similarly.

We know that $3 \theta^{ \pm}$are two arguments of $g_{a}(b)$. As $g_{a}(b) \in W_{0}$ and $\beta_{2}$, the point in $J_{a}$ with external angle $\frac{2}{3}$, is in $W_{2}$, we know that $\left(\frac{2}{3}, u\right),\left(3 \theta^{-}, u\right)$, $\left(3 \theta^{+}, u\right)$ are placed counterclockwise in $e^{u}$.

On the other hand, the points $\left(\frac{1}{3}, l\right),\left(\frac{1}{13}, l\right),\left(\frac{12}{13}, l\right)$ are placed clockwise in $e^{l}$.

Note that $\left(R_{a}\left(\frac{2}{3}\right) \cup R_{P_{1}}\left(\frac{1}{3}\right)\right) \cap N$ is a vertical segment connecting $\left(\frac{2}{3}, u\right)$ to $\left(\frac{1}{3}, l\right)$. Now we can modify $\gamma_{-}^{\prime} \cap N$ so that it consists of two affine segments disjoint from $\left(R_{a}\left(\frac{2}{3}\right) \cup R_{P_{1}}\left(\frac{1}{3}\right)\right) \cap N$ and connecting $\left(3 \theta^{-}, u\right)$ to $\left(\frac{1}{13}, l\right)$ and $\left(3 \theta^{+}, u\right)$ to $\left(\frac{12}{13}, l\right)$ respectively. This, together with $\gamma_{-}^{\prime}-N$, defines our Jordan curve $\gamma_{-}$. See Figure 6.

Turning to the modification of $\gamma_{+}^{\prime}$, we will use $\gamma_{-}$ as reference rather than $R_{a}\left(\frac{2}{3}\right) \cup R_{P_{1}}\left(\frac{1}{3}\right)$, which is no longer good. The points $\left(3 \theta^{-}, u\right),\left(3 \theta^{+}, u\right),\left(\theta^{-}, u\right)$, 
$\left(\theta^{+}, u\right)$ appear counterclockwise on $e^{u}$ and the points $\left(\frac{1}{13}, l\right),\left(\frac{12}{13}, l\right),\left(\frac{9}{13}, l\right),\left(\frac{4}{13}, l\right)$ appear clockwise on $e^{l}$. So we can modify $\gamma_{+}^{\prime} \cap N$ so that it consists of two affine segments disjoint from $\gamma_{-}$and connecting $\left(\theta^{-}, u\right)$ to $\left(\frac{9}{13}, l\right)$ and $\left(\theta^{+}, u\right)$ to $\left(\frac{4}{13}, l\right)$ respectively. This, together with $\gamma_{+}^{\prime}-N$, defines our Jordan curve $\gamma_{+}$. See Figure 6 .

By construction, $\gamma_{-}$and $\gamma_{+}$are disjoint; moreover one disc component $D_{+}$in the complement of $\gamma_{+}$contains only $a$ and $y$ as postcritical points, while a disc component $D_{-}$in the complement of $\gamma_{-}$contains only $F^{2}(w)$ and $z$ as postcritical points. Set $A_{0}=F^{-1}\left(D_{+}\right)$.

Note that $S^{2}-D_{+}$is a disc and that it contains $D_{-}$, the connected set

$$
s=\left[b, \beta_{0}\right] \cup R_{a}(0) \cup R_{P_{1}}(0) \cup\left[\beta^{\prime}, y_{*}\right],
$$

and a unique critical value, which is $z$. By looking at components of $F^{-1}(s)$ one can prove easily that $b_{1}, b_{2} \in \partial^{+} A_{0}$ (the component of $\partial A_{0}$ separating $A_{0}$ and $a$ ), the point $b_{0}$ is in the other component of $\partial A_{0}$ and $D_{+}$is homotopically contained in a component of $F^{-1}\left(D_{-}\right)$. Iterating backward once more we can show that $b_{10}, b_{20}$ are in the same component of $F^{-1}\left(\partial^{+} A_{0}\right)$.

Now the fact that $b_{0}, b_{1}, b_{2} \notin\left[g_{a}(a), \beta_{0}\right]$ implies that $A_{0}$ is essentially contained in the annulus $A$ bounded by $\gamma_{+}$an $\gamma_{-}$, and $b_{10}, b_{20} \notin\left[g_{a}(a), \beta_{0}\right]$ implies that the component of $F^{-1}\left(\partial^{+} A_{0}\right)$ containing $b_{10}$ is essentially contained in $A$. Now define $\Gamma$ to be the set of curves $\gamma$ such that $\gamma$ is essentially contained in $A-A_{0}$ and is homotopic to a curve of $F^{-n}\left(\gamma_{-}\right)$for some $n \geq 0$. By Proposition 2.3, $\Gamma$ is a nested obstruction and not a Levy cycle.
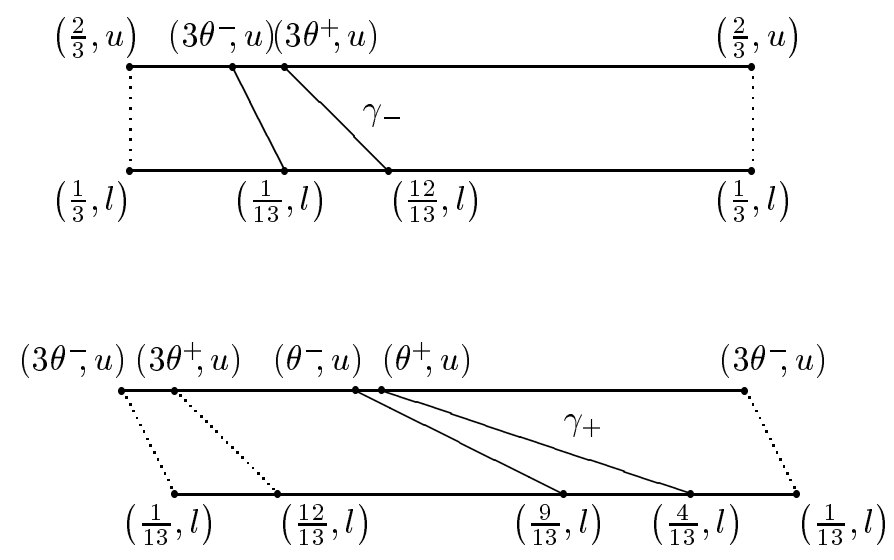

FIGURE 6. Modifications in $N$.
Proof of Theorem 2.4, $4 \Rightarrow 5$. There is a polynomial $g_{c}$ for which $w$ is three-periodic and $\theta^{ \pm}(c)=\frac{7}{26}, \frac{9}{26}$. Its Hubbard tree is the segment $[c, g(c)]$ in Figure 2 and the entropy is $\log \left(\frac{1}{2}(1+\sqrt{5})\right)$. By monotonicity of entropies (see [Douady 1995], for example), any $g_{a}$ with $\frac{7}{26} \sim_{g_{a}} \frac{9}{26}$ will have an entropy on its Hubbard tree at least $\log \left(\frac{1}{2}(1+\sqrt{5})\right)$.

Proof of Theorem 2.4, $2 \Rightarrow 5$. We will need the following (unpublished) result of M. Shishikura: Let $\Gamma$ be a Thurston obstruction for a mating $F=g \Perp f$. Then

$$
\mu_{\Gamma} \leq e^{h_{\mathrm{top}}\left(H_{g}\right)} .
$$

where $h_{\text {top }}\left(H_{g}\right)$ denotes the topological entropy of the map $g: H_{g} \rightarrow H_{g}$.

Now let $F=g_{a} \Perp P_{1}$ and $\Gamma^{\prime}$ be a nested obstruction. By Proposition 2.3, $\Gamma^{\prime}$ contains a subobstruction $\Gamma$ with $\mu_{\Gamma}=\frac{1}{2}(1+\sqrt{5})$. Put this into (5-2) we get

$$
e^{h_{\mathrm{top}}\left(H_{g}\right)} \geq \frac{1}{2}(1+\sqrt{5})
$$

which is Condition 5 of the theorem.

Proof of Theorem 2.4, $3 \Rightarrow 4$. Assume $a \in W_{1}$ (otherwise consider $\left.g_{\bar{a}}\right)$. If $g_{a}(a) \in W_{0}$ and $g_{a}^{3}$ is renormalizable, the small Julia set containing $a$ has a fixed point (for $g_{a}^{3}$ ) in $[a, w]$ with external angles $\frac{7}{26}, \frac{9}{26}$. If $g_{a}(a), g_{a}^{2}(a) \in W_{0}$, then $w_{0} \in\left[a, g_{a}(a)\right]$. Since $g_{a}$ maps each segment in the sequence

$$
\left[\alpha_{0}, w_{0}\right] \rightarrow[\alpha, w] \rightarrow[\alpha, a] \rightarrow\left[\alpha, g_{a}(a)\right]
$$

homeomorphically onto the next (Figure 5), and since $\left[\alpha, g_{a}(a)\right]$ contains $\left[\alpha_{0}, w_{0}\right]$, there is a 3 -periodic point $z$ in $\left[\alpha_{0}, w_{0}\right]$. An easy calculation shows that $g_{a}^{2}(z)$ has external angles $\frac{7}{26}, \frac{9}{26}$.

\section{APPENDIX: MATINGS SEEN IN PARAMETER SPACE AND SOME NUMERICAL OBSERVATIONS}

The rational maps in $\mathcal{A}$ form, up to Möbius transformation, a one-parameter family given by:

$$
\begin{aligned}
F_{t}(z) & =\frac{(3 t-2) z^{3}-\left(t^{4}-3 t^{2}+5 t-2\right)(3 z-2)}{(3 t-2) z^{3}-t^{3}(3 z-2)} \\
& =1-\frac{(t-1)^{3}(t+2)(3 z-2)}{(3 t-2) z^{3}-t^{3}(3 z-2)}
\end{aligned}
$$

with the parameter $t \in \mathbb{C}-\left\{-2, \frac{2}{3}, 1\right\}$. Here $0, \infty, 1, t$ correspond to $w, x, y, z$; in other words, 0 is a free 
double critical point, $\infty$ and 1 are two simple critical points, with orbit $\infty \mapsto 1 \mapsto t \mapsto \infty$.

Figure 7 shows the $t$-plane in the window $-5 \leq$ Re $t \leq 5,-5 \leq \operatorname{Im} t \leq 5$, and then again under magnification near the point $t=-2$.

We first describe several major hyperbolic components. The unique unbounded light grey component consists of $t$-values such that 0 is in the immediate basin of $\infty$ for $F_{t}$. The central dark grey component $G$ consists of $t$ values such that 0 is in the immediate basin of $t$ for $F_{t}$. The largest black component $B$ symmetric with respect to $\mathbb{R}$ represents $t$ values such that 0 is in the immediate basin of 1 for $F_{t}$. Note that $0 \in G$ and the degenerate parameter $t=1$ is in $B$. (In the zoom-in of Figure 8 the set $G$ touches the left edge and $B$ touches the right edge.)
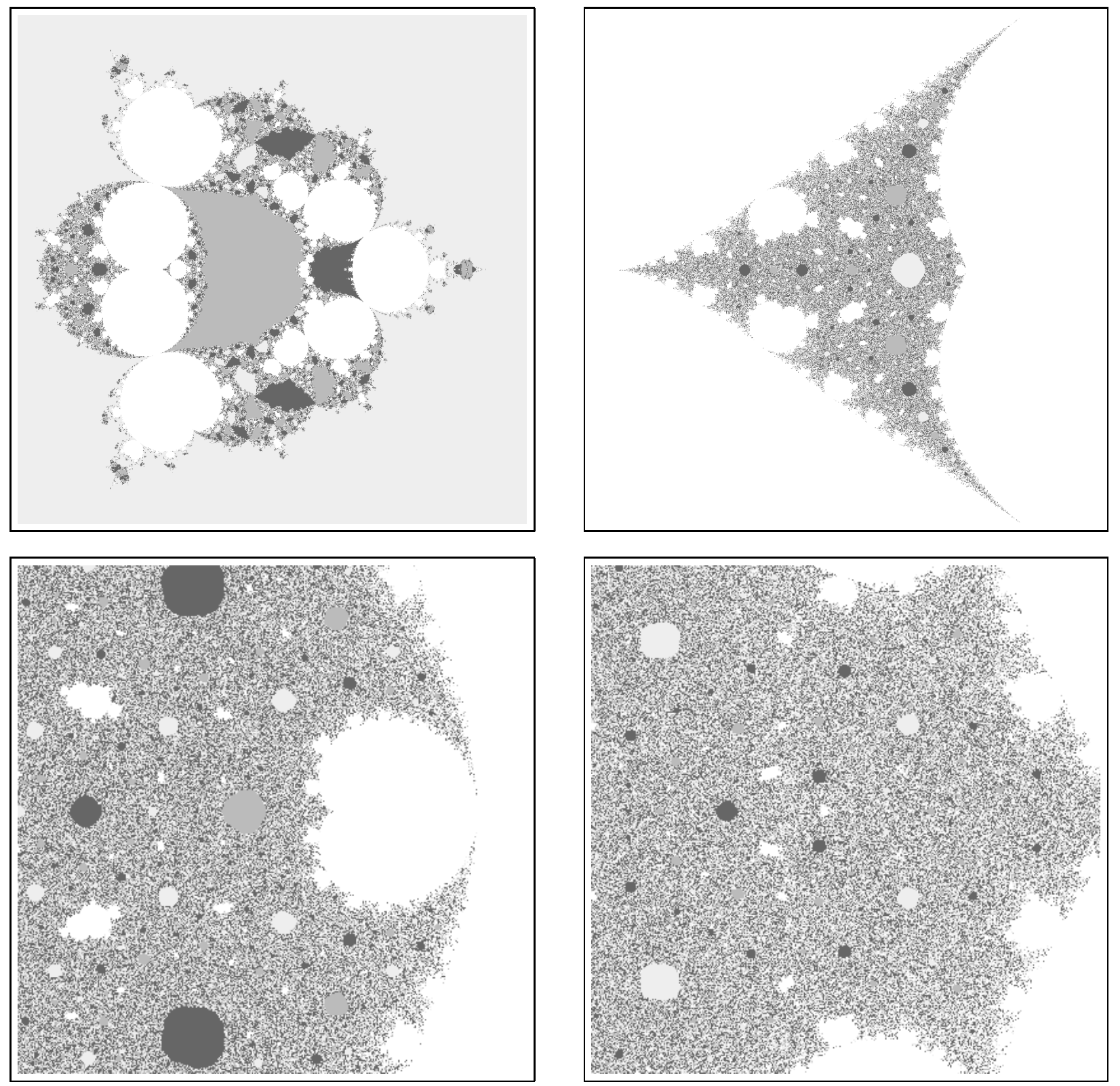

FIGURE 7. The family $\left\{F_{t}\right\}$. On the top left is the region $-5 \leq \operatorname{Re} t \leq 5,-5 \leq \operatorname{Im} t \leq 5$. The remaining panes zoom in at -2 with increasingly greater magnifications. In each case, white pixels represent values of $t$ for which the orbit $\left\{F_{t}^{n}(0)\right\}$ is not attracted by the orbit of $\infty$. We color a $t$-value light grey, dark grey, or black if $F_{t}^{3 p}(0)$, $F_{t}^{3 p+1}(0), F_{t}^{3 p+2}(0)$, respectively, is contained in the basin of $\infty$, for some integer $p \geq 0$. 


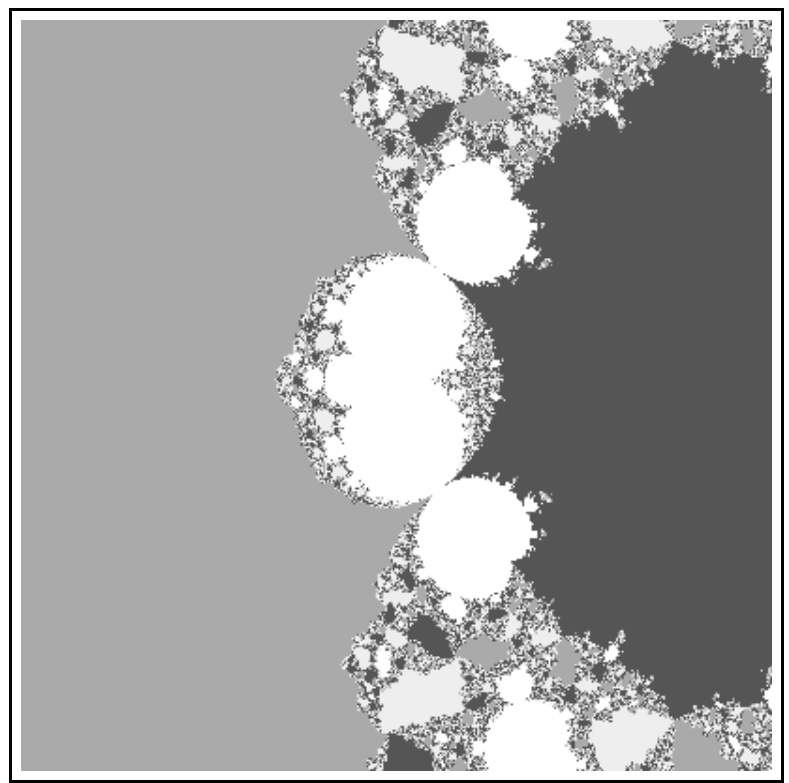

FIGURE 8. The family $\left\{F_{t}\right\}$ near the point $\frac{2}{3}$.

There are four "simplest" (but not always largest) white components, defined as follows.

There are four distinguished $t$-values for which 0 is fixed under $F_{t}$. They are the solutions of $t^{4}-$ $3 t^{2}+5 t-2=0$. Two of them, denoted $t_{1}, t_{2}$ with $t_{1}<t_{2}$, are real, and two, denoted by $t_{3}, t_{4}$ with $\mathrm{Imt}_{3}>0$, are complex conjugate. For $i=1,2,3,4$, the polynomial $F_{t_{i}}$ is conjugate to $P_{i}$.

Denote by $W_{i}$ the white component containing $t_{i}$, for $i=1,2,3,4$. Then $W_{1}$ is the largest white component on the left of $G$. The boundary of $W_{1}$ has two cusps, the right one corresponding to the left corner of Figure 7, top right. The set $W_{2}$ is the largest white component sitting between $G$ and $B$. Figure 8 gives a closer look of it. The boundary of $W_{2}$ has three cusps, with the unique real cusp at the degenerate parameter $t=\frac{2}{3}$. $W_{3}$ and $W_{4}$ are the two big white components above and below $B$. Their boundaries have two cusps.

We now give an interpretation of our result about matings. Define

$$
M_{3}=\left\{a \in \mathbb{C} \mid g_{a}^{n}(0) \not \rightarrow \infty \text { as } n \rightarrow \infty\right\}
$$

(see Figure 9) and

$$
M^{\prime}=\left\{a \in \mathbb{C} \mid g_{a} \text { is postcritically finite }\right\} \subset M_{3} .
$$

Recall that, for $i=1,2,3,4$, we defined

$$
A_{i}=\left\{a \in M^{\prime} \mid g_{a} \text { and } P_{i} \text { are matable }\right\} \text {. }
$$

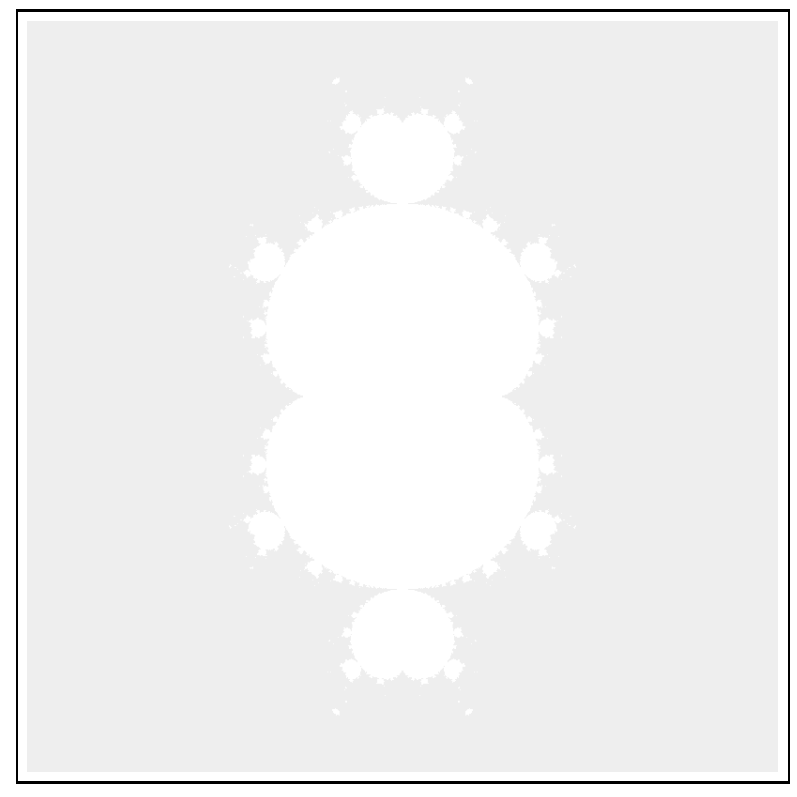

FIGURE 9. The connected locus of the family $z^{3}+a$.

For $a \in A_{i}$ the mating $g_{a} \Perp P_{i}$ is weakly equivalent to $F_{t(a, i)}$ for a unique $t=t(a, i)$ (Theorem 1.1 of Thurston). In particular, for $a=0$, we have $g_{0} \Perp P_{i} \sim F_{t_{i}}$ for $i=1,2,3,4$. We denote by $m_{i}$ the mapping $A_{i} \rightarrow \mathbb{C}, a \mapsto t(a, i)$. Therefore $m_{i}(0)=$ $t_{i} \in W_{i}$.

Theorem 2.6 shows that $A_{3}=A_{4}=M^{\prime}$. Denote by $L_{t}$ the limb of $M_{3}$ with internal angle $t$ and by $\gamma_{M_{3}}(\theta)$ the landing point in $M_{3}$ (if it exists) of the external ray of angle $\theta$. Then (as proved in the case of the Mandelbrot set) $L_{\frac{3}{4}}$ coincides with the set of $a \in M_{3}$ such that $\frac{5}{8} \sim_{g_{a}} \frac{7}{8}$.

Theorem 2.5 shows $A_{2} \subset M^{\prime}-L_{\frac{3}{4}}$ and we conjecture that $A_{2}=M^{\prime}-L_{\frac{3}{4}}$.

The set $A_{1}$ is dealt with by Theorem 2.2.

Similarly to the study of the Mandelbrot set, one can show that $\gamma_{M_{3}}\left(\frac{7}{26}\right)=\gamma_{M_{3}}\left(\frac{9}{26}\right)$, it is a root of a hyperbolic component $W(c)$ with center $c$ as in Section $2 \mathrm{C}$ and $\gamma_{M_{3}}\left(\frac{8}{26}\right)$ is the other root of $W(c)$.

Next, if we denote by $L_{\frac{7}{26}}, \frac{9}{26}$ the component of $M_{3}-\left\{\gamma_{M_{3}}\left(\frac{7}{26}\right), \gamma_{M_{3}}\left(\frac{9}{26}\right)\right\}$ containing $c$ and by $M(c)$ the small copy of $M_{3}$ centered at $c$, then $c \in W(c) \subset$ $M(c) \subset L_{\frac{7}{26}, \frac{9}{26}}$, the set $M(c)$ corresponds to the set of $a$ such that $g_{a}^{3}$ is renormalizable and $\frac{7}{26} \sim_{g_{a}} \frac{9}{26}$, and the set $L_{\frac{7}{26}, \frac{9}{26}}$ corresponds to the set of $a \in M_{3}$ such that $\frac{7}{26} \sim_{g_{a}} \frac{9}{26}$.

Furthermore, the union of the internal rays of $W(c)$ with angles 0 and $1 / 2$ separates $L_{\frac{7}{26}, \frac{9}{26}}$ into 
two halves, named by $L_{\frac{7}{26}}, \frac{8}{26}$ and $L_{\frac{8}{26}}, \frac{9}{26}$ respectively, with the last one containing $\gamma_{M_{3}}\left(\frac{1}{3}\right)$.

Condition 4 of Theorem 2.2 corresponds to

$$
L_{\frac{7}{26}, \frac{9}{26}} \cup L_{\frac{17}{26}, \frac{19}{26}}=L_{\frac{7}{26}, \frac{9}{26}} \cup \bar{L}_{\frac{7}{26}, \frac{9}{26}} .
$$

We conjecture that $A_{1}=M^{\prime}-L_{\frac{7}{26}, \frac{9}{26}} \cup L_{\frac{17}{26}}, \frac{19}{26}$. Condition 3 of Theorem 2.2 corresponds to

$$
M^{\prime} \cap\left(M(c) \cup \bar{M}(c) \cup L_{\frac{8}{26}, \frac{9}{26}} \cup L_{\frac{17}{26}, \frac{18}{26}}\right),
$$

and Theorem 2.2 shows that

$$
A_{1} \subset M^{\prime}-\left(M(c) \cup \bar{M}(c) \cup L_{\frac{8}{26}, \frac{9}{26}} \cup L_{\frac{17}{26}, \frac{18}{26}}\right) .
$$

Condition 5 is more difficult to describe. One can show, however, that any $a$ in the segment from 0 to $\gamma_{M_{3}}\left(\frac{7}{26}\right)$ has topological entropy smaller than $\log \left(\frac{1}{2}(1+\sqrt{5})\right)$, and therefore, by Theorem 2.2 , is in $A_{1}$.

Our computer observation strongly suggests that the mappings $m_{i}$ are continuous and can be extended to a considerably large and connected subset of $M_{3}$. Moreover it seems that $m_{4}\left(L_{\frac{7}{8}}\right)=m_{3}\left(L_{\frac{5}{8}}\right)$ and $m_{1}\left(L_{\frac{1}{4}}-L^{\prime}\right)=m_{1}\left(-\left(L_{\frac{1}{4}}-L^{\prime}\right)\right)$. Using shared mating techniques, we can actually show that

$$
\begin{aligned}
& m_{1}\left(L_{\frac{7}{8}} \cap M^{\prime}\right) \subset m_{3}\left(M^{\prime}\right), \\
& m_{2}\left(L_{\frac{7}{8}} \cap M^{\prime}\right) \subset m_{3}\left(M^{\prime}\right), \\
& m_{4}\left(L_{\frac{7}{8}} \cap M^{\prime}\right) \subset m_{3}\left(M^{\prime}\right) .
\end{aligned}
$$

The period-two hyperbolic component $D$ of $M_{3}$ attached to the main component $W_{0}$ with internal angle $\frac{3}{4}$ does not seem to have a corresponding image (under $m_{2}$ ) attached to $W_{2}$. We think that $t=\frac{2}{3}$ corresponds to the cutting point (or the root) of $D$ of $M_{3}$. This supports the conjecture that $A_{2}=$ $M^{\prime}-L_{\frac{3}{4}}$.

The degenerate parameter $t=-2$ is much more mysterious. The component $W_{1}$ looks very much like $W_{0}$. However there is a period 2 component $W_{1,2}$ which is attached to $W_{1}$ at two points, each corresponding to a root of $W_{1,2}$. This suggests that $W_{1,2}$ can be considered as a self shared mating, and can be indeed proved. The point $t=-2$ is surrounded by $W_{1,2}$ and $W_{1}$ (see the zoom-ins in Figure 7 ), and we see no white component attached to $t=-2$.

This is the first time in our experience that we see a singularity in an analytic family which is not on the boundary of any structurally stable component. We think that this should be related to the presence of non Levy cycle obstructions in $\mathcal{A}$.
There is another way to construct rational maps from polynomials, named by 'captures'. It has been intensively studied by B. Wittner, M. Rees, J. Head, Tan Lei and others. For the precise definition see [Tan 1997]. It is farely easy to show that, for a postcritically finite map $F_{t}$, if the basins of $x, y$ and $z$ are attached at a common fixed point, then $F_{t}$ is equivalent to either a mating or a capture. One may ask if this is still true for any postcritically finite $F_{t}$ (it is indeed the case for cubic Newton maps; see [Tan 1997]). Note that our family $\left\{F_{t}\right\}$ is somewhat similar to the family of quadratic rational maps with one critical point three-periodic (they both have one free critical point and a period-three orbit containing the remaining critical points). We therefore believe that, as known in the quadratic family due to Wittner, there are maps in the form of $F_{t}$ with $w$ periodic and not equivalent to a mating (they are by definition not equivalent to a capture).

\section{ACKNOWLEDGMENTS}

We express our thanks to J. H. Hubbard for his great attention to this research and many helpful discussions, to B. Bielefield and Y. Fisher for having helped us for the computer experiments, to B. Branner for having suggested us to study matings of cubic polynomials, and to Mary Rees for her help in greatly improving both our results about $g_{a} \Perp P_{1}$ and the global structure of the paper.

We also thank the Max-Planck-Institut für Mathematik in Bonn for having supported the first steps in this research and for having offered a friendly working atmosphere.

\section{REFERENCES}

[Bielefeld et al. 1992] B. Bielefeld, Y. Fisher, and J. Hubbard, "The classification of critically preperiodic polynomials as dynamical systems", J. Amer. Math. Soc. 5:4 (1992), 721-762.

[Carleson and Gamelin 1993] L. Carleson and T. W. Gamelin, Complex dynamics, Springer, New York, 1993.

[Douady 1995] A. Douady, "Topological entropy of unimodal maps: monotonicity for quadratic polynomials", pp. 65-87 in Real and complex dynamical systems (Hillerød, 1993), edited by B. Branner and P. Hjorth, Kluwer Acad. Publ., Dordrecht, 1995. 
[Douady and Douady 1979] R. Douady and A. Douady, Algèbre et théories galoisiennes, vol. 2, CEDIC, Paris, 1979 .

[Douady and Hubbard 1984] A. Douady and J. H. Hubbard, Étude dynamique des polynômes complexes, I, Publications d'Orsay 84-02, Université de ParisSud, Orsay, France, 1984.

[Douady and Hubbard 1985] A. Douady and J. H. Hubbard, Étude dynamique des polynômes complexes, II, Publications d'Orsay 85-04, Université de ParisSud, Orsay, France, 1985.

[Douady and Hubbard 1993] A. Douady and J. H. Hubbard, "A proof of Thurston's topological characterization of rational functions", Acta Math. 171:2 (1993), 263-297.

[Gantmacher 1959] F. R. Gantmacher, The theory of matrices, Chelsea, New York, 1959.

[Levy 1985] S. Levy, Critically finite rational maps, Ph.D. thesis, Princeton University, Princeton, NJ, 1985.

[Moore 1925] R. L. Moore, "Concerning upper semicontinuous collections of continua", Trans. Amer. Math. Soc. 27:4 (1925), 416-428.

[Pilgrim 1994] K. Pilgrim, Cylinders for iterated rational maps, Ph.D. thesis, University of California, Berkeley, 1994.
[Pilgrim and Tan 1998] K. M. Pilgrim and Tan L., "Combining rational maps and controlling obstructions", Ergodic Theory Dynam. Systems 18:1 (1998), 221245 .

[Poirier 1993] A. Poirier, "On post critically finite polynomials", preprint 1993/7, SUNY Stony Brook, 1993.

[Rees 1986] M. Rees, "Realization of matings of polynomials as rational maps of degree two", 1986. Manuscript.

[Rees 1992] M. Rees, "A partial description of parameter space of rational maps of degree two, I", Acta Math. 168:1-2 (1992), 11-87.

[Shishikura 2000] M. Shishikura, "On a theorem of M. Rees for the matings of polynomials", pp. 289-305 in The Mandelbrot set: theme and variations, edited by Tan Lei, London Math. Soc. lecture notes series 274, Cambridge Univ. Press, Cambridge, 2000.

[Tan 1992] Tan Lei, "Matings of quadratic polynomials", Ergodic Theory Dynamical Systems 12:3 (1992), 589620.

[Tan 1997] Tan Lei, "Branched coverings and cubic Newton maps", Fund. Math. 154:3 (1997), 207-260.

[Thurston 1983] W. Thurston, "The combinatorics of iterated rational maps", preprint, Princeton University, Princeton, NJ, 1983.

[Wittner 1986] B. Wittner, On the bifurcation loci of rational maps of degree two, Ph.D. thesis, Cornell University, Ithaca, NY, 1986.

Mitsuhiro Shishikura, Hiroshima University, Department of Mathematics, Kagamiyama, Higashi-Hiroshima 739-8526, Japan (mitsu@math.sci.hiroshima-u.ac.jp)

Tan Lei, Département de Mathématiques, Université de Cergy-Pontoise, 2 Avenue Adolphe Chauvin, 95302 Cergy-Pontoise, France (tanlei@math.u-cergy.fr)

Received March 19, 1998; accepted in revised form January 13, 1999 